\title{
THE EFFECTS OF SMALL-SIDED SOCCER GAMES ON TECHNICAL ACTIONS AND SKILLS: A SYSTEMATIC REVIEW
}

review paper

(c) University School of Physical Education in Wroclaw

DOI: https://doi.org/10.5114/hm.2020.93014

\section{FILIPE MANUEL CLEMENTE ${ }^{1,2}$, HUGO SARMENTO ${ }^{3}$}

${ }^{1}$ Escola Superior de Desporto e Lazer, Instituto Politécnico de Viana do Castelo, Viana do Castelo, Portugal

${ }^{2}$ Instituto de Telecomunicações, Delegação da Covilhã, Covilhã, Portugal

${ }^{3}$ Research Unit for Sport and Physical Activity, Faculty of Sports Sciences and Physical Education,

University of Coimbra, Coimbra, Portugal

\section{ABSTRACT}

Purpose. This systematic review aims to describe evidence of the effects of different small-sided soccer games (SSGs) on technical actions and technical performance. The article systematically reviews and organizes the effects of the most common task conditions and characterizes the methodologies employed in previous studies.

Methods. A systematic review of Web of Science, PubMed, and SPORTDiscus databases was conducted in accordance with preferred reporting items for systematic reviews and meta-analyses (PRISMA) guidelines. The search returned 339 records. After screening against the set criteria, a total of 37 full articles were fully reviewed.

Results. The main topics related to consequences of technical actions were (1) effects of different formats; (2) effects of different pitch sizes, relative area per player, pitch restrictions and configurations; (3) effects of different task conditions; (4) effects of age group, experience, and technical skill; and (5) effects of different training regimens or structured training programs. Briefly, it was concluded that smaller formats meaningfully increased the number of determinant technical actions performed when compared with medium and larger formats (5 vs. 5 to 11 vs. 11). Furthermore, smaller amounts of relative area per player meaningfully increased most technical actions made by players. The use of free play increased the number of technical actions taken, while imposing a 1-touch limitation raised the number of involvements but also the frequencies of errors and balls lost.

Conclusions. This systematic review reveals that each of the above-mentioned topics has a meaningful impact on the frequencies of technical actions and success during SSGs.

Key words: association football, performance, drill-based games, conditioned games, small-sided games, skills

\section{Introduction}

Small-sided games (SSGs) are small and conditioned versions of the formal format of play and have been progressively growing in terms of their application and related research in soccer [1, 2]. These games apply to training contexts mainly because they allow the perceptions of players to be augmented for specific behaviours [3]. Moreover, it is also possible at the same time to develop tactical behaviours and technical actions, providing consequences in psychobiological responses during games [4]. Such multidimen- sional effects of SSGs on players' acute responses make these games highly popular and often used by coaches for players of different age groups, experience levels, and competitive levels [5, 6].

The representativeness of SSGs, considering the formal game format, allows the chaotic effect of soccer to be simplified without compromising the essential characteristics of the game or its dynamic and complex nature [7]. In fact, SSGs allow players to repeat without repeating. That is, players are exposed to given task conditions that augment their perceptions of specific behaviours without having to repeat

Correspondence address: Filipe Manuel Clemente, Complexo Desportivo e Lazer de Melgaço - Monte de Prado, 4960-320, Melgaço, Portugal, e-mail: filipe.clemente5@gmail.com

Received: November 10, 2019

Accepted for publication: February 6, 2020

Citation: Clemente FM, Sarmento H, The effects of small-sided soccer games on technical actions and skills: a systematic review. Hum Mov. 2020;21(3):100-119; doi: https://doi.org/10.5114/hm.2020.93014. 
mechanical actions, which is often the case in analytical training (i.e., skill-based drills) [8]. Naturally, because the dynamics of the game is maintained during SSGs, the games influence the emergence of different adaptive behaviours during the game, thus leading to a low-to-moderate reproducibility of the technical actions performed during SSGs [5]. Nevertheless, it is expected that changes in different task conditions may have different effects on players' technical performance.

Different conditions can be used in SSGs to adjust tasks to meet the coach's objectives and the players' needs. However, conditions should be manipulated carefully. In research on SSGs, the following variables are commonly manipulated [1]: (1) format of play (i.e., the number of players involved and the numerical relationship between teams); (2) pitch size (i.e., the width and length of the pitch and their relationship, the relative mean area per player, and the restrictions or configurations of the pitch); (3) goal characteristics (i.e., the use of either formal or smaller goals with or without goalkeepers and the use of different scoring methods); (4) rule modifications (e.g., limiting the number of consecutive touches on the ball allowed; using or not using the offside rule; the use of different types of defensive markings); (5) training regimen (i.e., the work-to-rest relationship); and (6) coach encouragement (i.e., the verbal stimulation of coaches during SSGs). However, other possible task constraints have also been researched during SSGs, namely the influences of age, skill level, tactical knowledge, physical status, or mental fatigue on players [9-11].

The effects of these conditions/constraints on players' psychobiological responses [2, 12, 13], tactical behaviours [14], and their effects at a multidimensional level $[1,15]$ have been summarized and systematically revised over the recent several years. However, there is a lack of systematization of the effects of SSGs on technical actions in male soccer players from different age categories. The systematization of such effects might help sports scientists and coaches to understand the main effects of different task conditions/ constraints on technical actions and could provide useful information that allows coaches to optimize the adjustment of the game to the purpose. As such, the purpose of this study was to systematically review and organize the literature on soccer SSGs to identify the effects of different task conditions on the technical actions performed by male players of different ages.

\section{Material and methods}

Search strategy: databases and inclusion criteria

The present systematic review and the associated search followed the PRISMA (preferred reporting items for systematic reviews and meta-analyses) guidelines [16]. The electronic databases of Web of Science, PubMed, and SPORTDiscus were searched on October $12^{\text {th }}, 2019$ for relevant publications, with the use of the keywords 'football' OR 'soccer,' each associated with 'SSG*' OR 'drill based training' OR 'small sided and conditioned games' OR 'position games' OR 'small sided' 'small-sided games'.

The inclusion criteria for the articles were: (1) relevant data concerning technical actions; (2) participants included any age group or competitive level; (3) only men were included; (4) only studies conducted in soccer players; (5) only studies published in English; and (6) only full articles. The following exclusion criteria were applied: (1) studies in women; (2) studies conducted in physical education contexts; (3) non-English articles; (4) conference abstracts, letters to the editor, errata, narrative reviews, systematic reviews, meta-analyses, or invited commentaries; (5) studies that did not include relevant data for this study or aroused serious concerns about the methodology; and (6) studies without analysis of technical actions.

Two reviewers independently screened citations and abstracts to identify articles potentially meeting the inclusion criteria. For those cases, the full article was retrieved and independently screened by the 2 reviewers to determine whether it met the inclusion criteria. Disagreements between the independent reviewers in terms of inclusion criteria were resolved through discussion.

\section{Quality of the studies and extraction of data}

The quality of the studies was assessed by a riskof-bias quality form of 16 items validated and adjusted for the specific context of SSG research $[1,17]$. The items of analysis were [1]: purpose (item 1), relevance of background literature (item 2), appropriateness of the study design (item 3), sample included (items 4 and 5), informed consent procedure (item 6), outcome measures (items 7 and 8), method description (item 9), significance of results (item 10), analysis (item 11), practical importance (item 12), description of drop-outs (item 13), conclusions (item 14), practical implications (item 15), and limitations (item 16). 
F. Clemente, H. Sarmento, Small-sided games and technical actions: a review

All the 16 quality criteria were scored on a binary scale (0/1), wherein 2 of those criteria (items 6 and 13) presented the option: 'If not applicable, assume NA'. Two independent reviewers screened and rated the included full articles and the scores were tested by using the $k$ agreement rate between reviewers. The result of $k$ agreement was $k=0.94$.

The sum of scores of all items was divided by the number of relevant scored items for the specific research design. On the basis of this procedure, the articles were classified as displaying [1]: (1) low methodological quality ( $\leq 50 \%$ ); (2) good methodological quality (51-75\%); and (3) excellent methodological quality (> 75\%).

A data extraction sheet [18] was used in the present systematic review to define the inclusion requirements and then tested with 10 randomly selected studies (pilot test). One author extracted the data, and the other verified them. Disagreements were resolved in discussions between the authors.

\section{Ethical approval}

The conducted research is not related to either human or animal use.

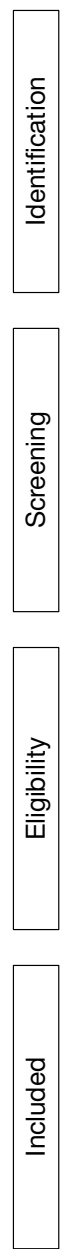

\section{Results}

Search, selection, and inclusion of publications

The initial search identified 339 titles in the aforementioned databases. These data were then exported to reference manager software (EndNote ${ }^{\mathrm{TM}} \mathrm{X} 8$, Clarivate Analytics, Philadelphia, USA). Any duplicates (199 references) were eliminated either automatically or manually. The remaining 140 articles were then screened for relevance on the basis of their title and abstract, which resulted in 41 studies eliminated from the database. The full text of the remaining 99 articles was examined in more detail; 62 were rejected because they did not meet the inclusion criteria. At the end of the screening procedure, 37 articles were selected for in-depth reading and analysis (Figure 1). The main factors for exclusion were that studies only tested tactical behaviour/collective organization $(n=$ 28 ) and only included analysis of internal and external load $(n=16)$. Other studies were excluded because they referred to formal games $(n=4)$; constituted conference abstracts $(n=4)$, narrative reviews $(n=2)$, or qualitative analyses $(n=2)$; were not written in English $(n=2)$, not related with SSGs $(n=2)$, related with other sports $(n=1)$, or related with fitness only $(n=1)$. Among the included studies, 38\% were published in the recent 3 years (i.e., 2017, 2018, and 2019).

Figure 1. PRISMA flowchart

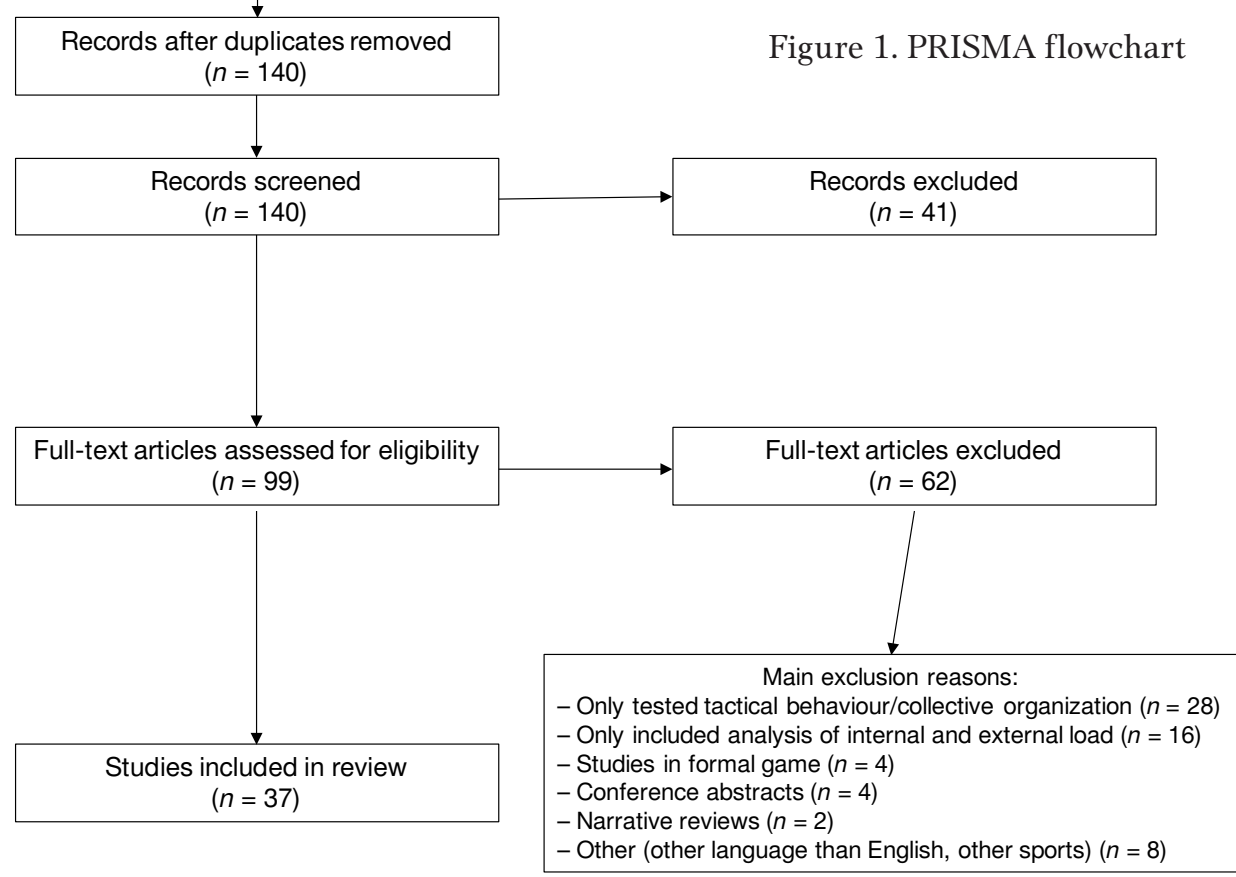




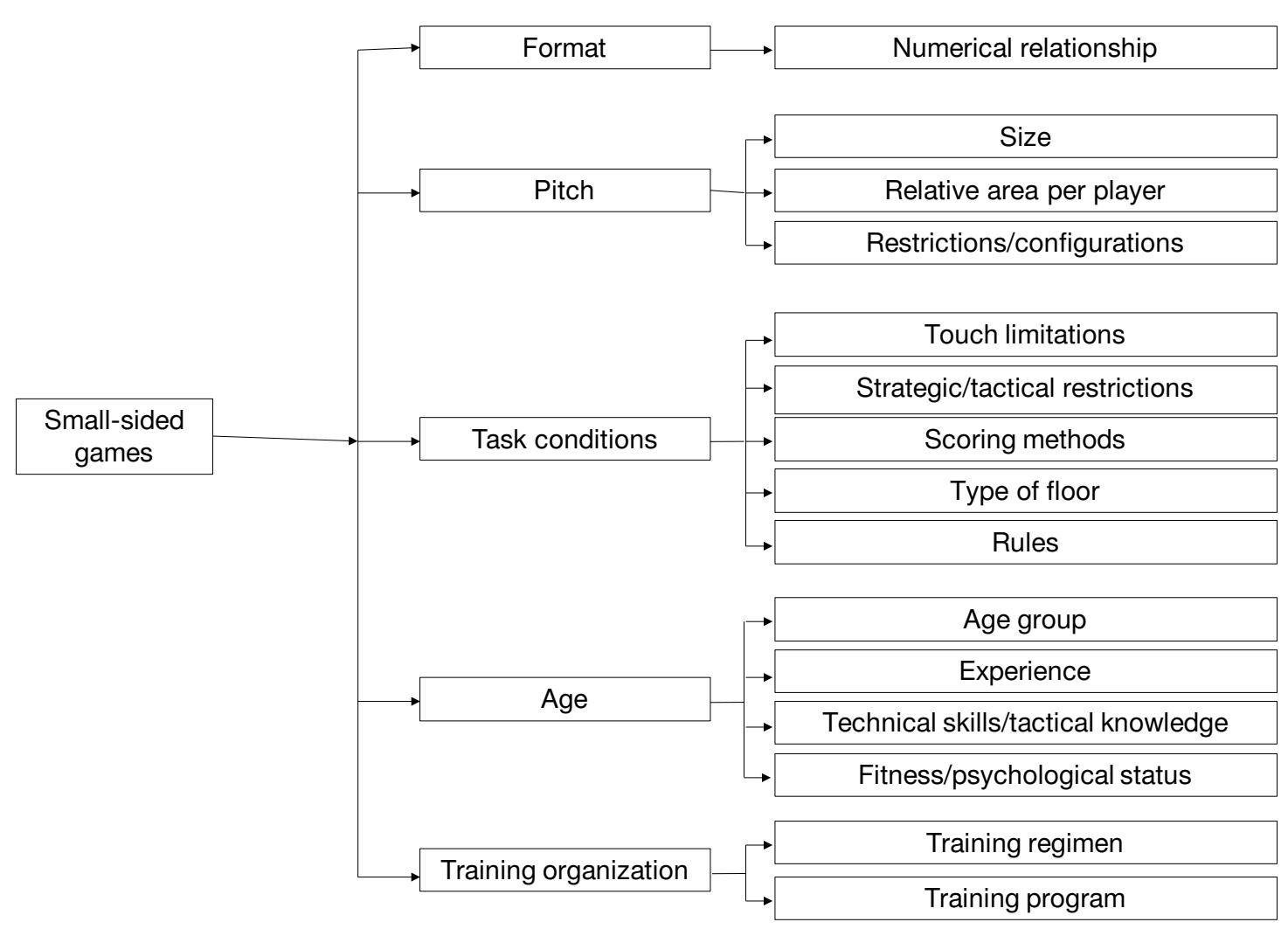

Figure 2. Main small-sided game topics

\section{Quality assessment}

None of the articles presented a $100 \%$ score, although the minimum score was $86.7 \%$. The main potential deficiencies of the 37 included papers were mainly related to failing to clearly acknowledge the study limitations and the justification for the sample size used.

\section{Data organization}

The effects of SSGs on technical actions were fundamentally related with manipulations of task conditions. On the basis of the in-depth reading and careful analysis, it was decided that the most appropriate way to present the results would be to organize the task condition in the following topics: (1) effects of different formats $(n=10)$; (2) effects of different pitch sizes, relative area per player, pitch restrictions and configurations ( $n=9)$; (3) effects of different task conditions ( $n=9)$; (4) effects of age group, experience, and technical skill $(n=4)$; and (5) effects of training regimens or structured training programs $(n=5)$. The specific organization in topics can be found in Figure 2 .

The 2 independent reviewers (FMC and HS) classified the articles depending on the main purpose of research and distributed in the research topics. Disagreements were resolved through discussion until a consensus was found.

\section{Effects of format}

Five studies [19-23] tested 1 vs. 1 to 3 vs. 3 SSGs, and 3 studies [24-26] included comparisons with games that followed the formal format. Two studies $[27,28]$ assessed the effects of unbalanced numerical relationships by using floaters (i.e., neutral players who provided support to the team via momentary possession of the ball) (Table 1 ).

Effects of pitch size, relative area per player, pitch restrictions and configurations

Seven of the 9 included studies [29-35] investigated the effects of different pitch dimensions and relative areas per player on technical actions made during different formats of play. One study [36] tested the manipulation of the width-to-length ratio. One paper [37] focused on the effects of different external markings on the pitch. One of the included articles [34] evaluated the effects of different pitch dimensions on actions performed by goalkeepers (Table 2). 
Table 1. Effects of different formats and numerical relationships on technical actions

\begin{tabular}{|c|c|c|c|c|c|c|c|c|c|c|}
\hline 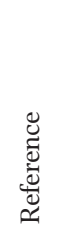 & 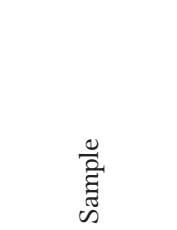 & 竞 & $\begin{array}{l}\text { हैँ } \\
\text { हैं } \\
\infty \\
\infty \\
\infty\end{array}$ & 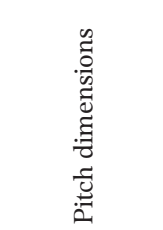 & 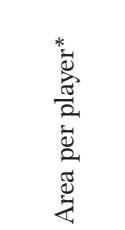 & 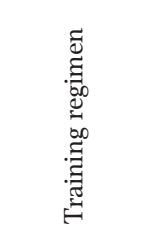 & $\frac{\mathscr{Q}}{3}$ & 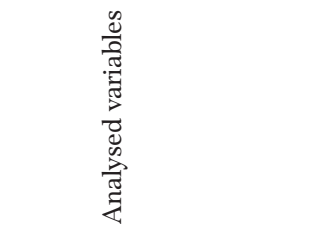 & 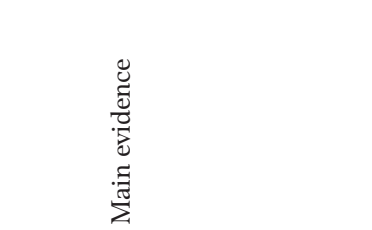 & 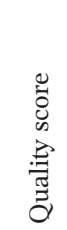 \\
\hline [19] & $\begin{array}{c}10 \text { soccer } \\
\text { players, } \\
13.5 \pm 0.5 \text { yo }\end{array}$ & $\begin{array}{l}\text { Analyse } \\
\text { the impact } \\
\text { of changes } \\
\text { in format } \\
\text { on technical } \\
\text { actions }\end{array}$ & $\begin{array}{l}3 \text { vs. } 3 \\
4 \text { vs. } 4 \\
5 \text { vs. } 5\end{array}$ & $\begin{array}{l}30 \times 30 \mathrm{~m} \\
30 \times 30 \mathrm{~m} \\
30 \times 30 \mathrm{~m}\end{array}$ & $\begin{array}{c}150 \mathrm{~m}^{2} \\
112.5 \mathrm{~m}^{2} \\
90 \mathrm{~m}^{2}\end{array}$ & $\begin{array}{l}3 \times 4^{\prime} / 3 \\
\text { active } \\
\text { recovery }\end{array}$ & $\begin{array}{l}\text { No goalkeepers; } \\
\text { small goals; goals } \\
\text { only considered } \\
\text { when all the } \\
\text { teammates were } \\
\text { in the opponent's } \\
\text { half of the pitch }\end{array}$ & $\begin{array}{l}\text { Involvements with the ball } \\
\text { Passes } \\
\text { Target passes } \\
\text { Crosses } \\
\text { Shot on goal } \\
\text { Tackles } \\
\text { Headers }\end{array}$ & $\begin{array}{l}\text { No meaningful changes in the } \\
\text { frequency of technical actions } \\
\text { were found between formats. } \\
\text { Variability of technical actions } \\
\text { varied between } 6.8 \text { and } 19.3 \% \\
\text { (CV). Maturation had no } \\
\text { influence on technical actions }\end{array}$ & 93.3 \\
\hline [20] & $\begin{array}{c}15 \text { soccer } \\
\text { players, } \\
26.3 \pm 4.9 \text { yo } \\
\text { (professional } \\
\text { players) }\end{array}$ & $\begin{array}{l}\text { Analyse } \\
\text { the impact } \\
\text { of changes } \\
\text { in format } \\
\text { on technical } \\
\text { actions }\end{array}$ & $\begin{array}{l}3 \text { vs. } 3+\mathrm{GK} \\
9 \text { vs. } 9+\mathrm{GK}\end{array}$ & $\begin{array}{l}30 \times 25 \mathrm{~m} \\
60 \times 50 \mathrm{~m}\end{array}$ & $\begin{array}{l}125 \mathrm{~m}^{2} \\
167 \mathrm{~m}^{2}\end{array}$ & $\begin{array}{c}3 \times 5^{\prime} / 4^{\prime} \\
\text { passive } \\
\text { recovery }\end{array}$ & $\begin{array}{l}\text { Normal match } \\
\text { rules }\end{array}$ & $\begin{array}{l}\text { Block } \\
\text { Dribble } \\
\text { Header } \\
\text { Interception } \\
\text { Pass } \\
\text { Receive } \\
\text { Shot } \\
\text { Turn } \\
\text { Tackle } \\
\text { Total ball contacts per game } \\
\text { Ball contacts per individual }\end{array}$ & $\begin{array}{l}\text { Meaningfully greater values } \\
\text { of block, header, interception, } \\
\text { pass, receive, turn, and total } \\
\text { ball contacts per game were } \\
\text { found in the } 9 \text { vs. } 9+\mathrm{GK} \text {. } \\
\text { Meaningfully greater number } \\
\text { of ball contacts per player, } \\
\text { dribbles, shots, and tackles } \\
\text { were found in the } 3 \text { vs. } 3+\mathrm{GK} \\
\end{array}$ & 86.7 \\
\hline [24] & $\begin{array}{c}40 \text { soccer } \\
\text { players, } \\
25.3 \pm 2.4 \text { yo } \\
\text { (international } \\
\text { players) }\end{array}$ & $\begin{array}{l}\text { Analyse } \\
\text { the impact } \\
\text { of changes } \\
\text { in format } \\
\text { on technical } \\
\text { actions }\end{array}$ & $\begin{array}{c}4 \text { vs. } 4 \\
\text { Match-play }\end{array}$ & $\begin{array}{c}30 \times 20 \mathrm{~m} \\
100 \times 60 \mathrm{~m}\end{array}$ & $\begin{array}{r}75 \mathrm{~m}^{2} \\
300 \mathrm{~m}^{2}\end{array}$ & $\begin{array}{c}4 \times 4 \text { ' } / 3 \\
\text { passive } \\
\text { recovery; } \\
90 \text { ' for the } \\
\text { match play }\end{array}$ & $\begin{array}{l}\text { There were } \\
3 \text { conditions on } \\
\text { the SSG (1-ball } \\
\text { touch limitation, } \\
\text { 2-ball touch } \\
\text { limitation, and } \\
\text { free play). The } \\
\text { game was played } \\
\text { aiming to keep the } \\
\text { ball possession } \\
\text { using } 4 \text { external } \\
\text { support players }\end{array}$ & $\begin{array}{l}\text { Number of duels } \\
\text { Percentage of successful } \\
\text { passes } \\
\text { Total number of balls lost } \\
\text { Total number of ball } \\
\text { possessions }\end{array}$ & $\begin{array}{l}\text { Greater number of duels } \\
\text { and lost balls and a lower } \\
\text { percentage of successful } \\
\text { passes and total number } \\
\text { of ball possessions were } \\
\text { observed in the } 4 \text { vs. } 4 \\
\text { compared with match-play. } \\
\text { Forwards had similar results } \\
\text { between SSG and match play }\end{array}$ & 86.7 \\
\hline [21] & $\begin{array}{c}20 \text { soccer } \\
\text { players, } \\
27 \pm 2 \text { yo } \\
\text { (international } \\
\text { players) }\end{array}$ & $\begin{array}{l}\text { Analyse } \\
\text { the impact } \\
\text { of changes } \\
\text { in format } \\
\text { and periods } \\
\text { on technical } \\
\text { actions }\end{array}$ & $\begin{array}{l}2 \text { vs. } 2 \\
3 \text { vs. } 3 \\
4 \text { vs. } 4\end{array}$ & $\begin{array}{l}20 \times 15 \mathrm{~m} \\
25 \times 18 \mathrm{~m} \\
30 \times 20 \mathrm{~m}\end{array}$ & $\begin{array}{l}75 \mathrm{~m}^{2} \\
75 \mathrm{~m}^{2} \\
75 \mathrm{~m}^{2}\end{array}$ & $\begin{array}{l}4 \times 2^{\prime} / 3^{\prime} \\
4 \times 3^{\prime} / 3^{\prime} \\
4 \times 4^{\prime} / 3^{\prime}\end{array}$ & $\begin{array}{l}\text { Two-ball touch } \\
\text { limitation. } \\
\text { The games were } \\
\text { played aiming } \\
\text { to keep the ball } \\
\text { possession using } \\
4 \text { external support } \\
\text { players }\end{array}$ & $\begin{array}{l}\text { Total number of duels } \\
\text { Successful passes (\%) } \\
\text { Total number of lost balls } \\
\text { Total number of ball } \\
\text { possessions }\end{array}$ & $\begin{array}{l}\text { Significantly greater number } \\
\text { of duels and percentage } \\
\text { of successful passes were } \\
\text { found in the first and second } \\
\text { periods (bouts) compared } \\
\text { with the last period (bout). } \\
\text { Frequency of balls lost increased } \\
\text { progressively from period } 1 \\
\text { to period } 4 \text {. Ball possessions } \\
\text { were similar across the periods }\end{array}$ & 86.7 \\
\hline [22] & $\begin{array}{c}10 \text { soccer } \\
\text { players, } \\
\text { under-10 (age } \\
\text { not described) }\end{array}$ & $\begin{array}{l}\text { Analyse } \\
\text { the impact } \\
\text { of changes } \\
\text { in format } \\
\text { and periods } \\
\text { on technical } \\
\text { actions }\end{array}$ & $\begin{array}{l}3 \text { vs. } 3+\mathrm{GK} \\
5 \text { vs. } 5+\mathrm{GK}\end{array}$ & $\begin{array}{l}36 \times 27 \mathrm{~m} \\
60 \times 45 \mathrm{~m}\end{array}$ & $\begin{array}{l}162 \mathrm{~m}^{2} \\
270 \mathrm{~m}^{2}\end{array}$ & $1 \times 8^{\prime}$ & Not mentioned & $\begin{array}{l}\text { Shoot at goal } \\
\text { Keep possession of the ball } \\
\text { Earn a foul, win a corner } \\
\text { or throw-in } \\
\text { Commit a foul, give away } \\
\text { a corner or throw-in } \\
\text { Loss of ball possession } \\
\text { Regain ball possession } \\
\text { Ball possession of the } \\
\text { opponent } \\
\text { Take a shot at own goal }\end{array}$ & $\begin{array}{l}\text { Shoots at goal and take } \\
\text { a shoot at own goal were } \\
\text { significantly greater } \\
\text { at the } 3 \text { vs. } 3\end{array}$ & 80.0 \\
\hline [25] & $\begin{array}{c}10 \text { soccer } \\
\text { players, } \\
27.6 \pm 4.1 \text { yo } \\
\text { (professional) }\end{array}$ & $\begin{array}{l}\text { Analyse } \\
\text { the impact } \\
\text { of changes } \\
\text { in format } \\
\text { and periods } \\
\text { on technical } \\
\text { actions }\end{array}$ & $\begin{array}{l}4 \text { vs. } 4 \\
5 \text { vs. } 5 \\
6 \text { vs. } 6 \\
7 \text { vs. } 7 \\
8 \text { vs. } 8 \\
9 \text { vs. } 9 \\
10 \text { vs. } 10 \\
11 \text { vs. } 11\end{array}$ & $\begin{aligned} 30 & \times 25 \mathrm{~m} \\
46 & \times 40 \mathrm{~m} \\
50 & \times 44 \mathrm{~m} \\
54 & \times 45 \mathrm{~m} \\
60 & \times 50 \mathrm{~m} \\
70 & \times 56 \mathrm{~m} \\
80 & \times 70 \mathrm{~m} \\
100 & \times 74 \mathrm{~m}\end{aligned}$ & $\begin{array}{r}94 \mathrm{~m}^{2} \\
184 \mathrm{~m}^{2} \\
183 \mathrm{~m}^{2} \\
174 \mathrm{~m}^{2} \\
188 \mathrm{~m}^{2} \\
218 \mathrm{~m}^{2} \\
280 \mathrm{~m}^{2} \\
336 \mathrm{~m}^{2}\end{array}$ & $\begin{array}{c}3 \times 5^{\prime} / 3^{\prime} \\
\text { rest }\end{array}$ & $\begin{array}{l}\text { Normal match } \\
\text { rules }\end{array}$ & $\begin{array}{l}\text { Pass } \\
\text { Receive } \\
\text { Turn } \\
\text { Dribble } \\
\text { Header } \\
\text { Tackle } \\
\text { Block } \\
\text { Interception } \\
\text { Shot }\end{array}$ & $\begin{array}{l}\text { The more players on the pitch, } \\
\text { the fewer total passes and } \\
\text { receives made. Significantly } \\
\text { greater number of dribbles } \\
\text { and shots were found in the } \\
\text { smaller formats. Greater } \\
\text { number of headings and } \\
\text { interceptions were found the } \\
\text { in the larger games }\end{array}$ & 86.7 \\
\hline
\end{tabular}




\begin{tabular}{|c|c|c|c|c|c|c|c|c|c|c|}
\hline [23] & $\begin{array}{l}18 \text { soccer } \\
\text { players, } \\
\text { under- } 11 \\
\text { (age not } \\
\text { described) }\end{array}$ & $\begin{array}{l}\text { Analyse } \\
\text { the impact } \\
\text { of changes } \\
\text { in format } \\
\text { on technical } \\
\text { actions }\end{array}$ & $\begin{array}{l}3 \text { vs. } 3+\text { GK } \\
6 \text { vs. } 6+\text { GK }\end{array}$ & $\begin{array}{c}30 \times 19.5 \mathrm{~m} \\
60 \times 39 \mathrm{~m}\end{array}$ & $\begin{array}{r}98 \mathrm{~m}^{2} \\
195 \mathrm{~m}^{2}\end{array}$ & $1 \times 8^{\prime}$ & Not mentioned & $\begin{array}{l}\text { Shoot at goal } \\
\text { Keep possession } \\
\text { of the ball } \\
\text { Earn a foul, win a corner } \\
\text { or throw-in } \\
\text { Commit a foul, give away } \\
\text { a corner or throw-in } \\
\text { Loss of ball possession } \\
\text { Regain ball possession } \\
\text { Ball possession of the } \\
\text { opponent } \\
\text { Take a shot at own goal }\end{array}$ & $\begin{array}{l}\text { Significantly greater values } \\
\text { of shoots at goal were found } \\
\text { in } 3 \text { vs. } 3 \text { format. Significantly } \\
\text { greater values of keep } \\
\text { possession of the ball, loss } \\
\text { of ball possession, and ball } \\
\text { possession of the opponent } \\
\text { were found in the } 6 \text { vs. } 6\end{array}$ & 80.0 \\
\hline [27] & $\begin{array}{l}44 \text { soccer } \\
\text { players, } \\
23.1 \pm 0.7 \text { yo } \\
\quad \text { (amateur; } \\
\quad n=22) \\
25.6 \pm 4.9 \text { yo } \\
\text { (professional; } \\
\quad n=22 \text { ) }\end{array}$ & $\begin{array}{l}\text { Analyse } \\
\text { the impact } \\
\text { of changes } \\
\text { in format } \\
\text { on technical } \\
\text { actions }\end{array}$ & $\begin{array}{l}4 \text { vs. } 3+\text { GK } \\
4 \text { vs. } 5+\text { GK } \\
4 \text { vs. } 7+\text { GK }\end{array}$ & $40 \times 30 \mathrm{~m}$ & $\begin{array}{l}171 \mathrm{~m}^{2} \\
133 \mathrm{~m}^{2} \\
109 \mathrm{~m}^{2}\end{array}$ & $1 \times 3^{\prime}$ & $\begin{array}{l}\text { Regular rules. } \\
\text { Scoreboard return } \\
\text { to } 0 \text { every time } \\
\text { when a difference } \\
\text { of } 2 \text { goals was } \\
\text { achieved }\end{array}$ & $\begin{array}{l}\text { Run to the ball } \\
\text { Wait } \\
\text { Control } \\
\text { Pass } \\
\text { Shoot } \\
\text { Protect } \\
\text { Drive } \\
\text { Feint } \\
\text { Dribble } \\
\text { Intercept } \\
\text { Deflect } \\
\text { Clear } \\
\text { Anticipate } \\
\text { Support } \\
\text { Unmark } \\
\text { Press } \\
\text { Delay } \\
\text { Dissuade } \\
\text { Balance } \\
\text { Withdraw }\end{array}$ & $\begin{array}{l}\text { Greater number of opponents } \\
\text { led to increases in the frequency } \\
\text { of defensive patterns (namely, } \\
\text { controlling) and a decrease } \\
\text { in the number of waiting. } \\
\text { Increasing the number of } \\
\text { opponents decreased the } \\
\text { number of passes and driving } \\
\text { by players with possession }\end{array}$ & 86.7 \\
\hline [28] & $\begin{array}{c}22 \text { soccer } \\
\text { players, } \\
17.2 \pm 0.9 \text { yo }\end{array}$ & $\begin{array}{l}\text { Analyse } \\
\text { the impact } \\
\text { of floaters } \\
\text { (internal } \\
\text { and external) } \\
\text { on technical } \\
\text { actions }\end{array}$ & 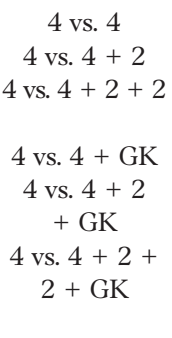 & $40 \times 30 \mathrm{~m}$ & $\begin{array}{r}150 \mathrm{~m}^{2} \\
120 \mathrm{~m}^{2} \\
\# 120 \mathrm{~m}^{2} \\
150 \mathrm{~m}^{2} \\
120 \mathrm{~m}^{2} \\
\# 120 \mathrm{~m}^{2}\end{array}$ & $4 \times 4^{\prime} / 2^{\prime}$ & $\begin{array}{l}\text { SSGs with } 2+2 \\
\text { neutral players } \\
\text { representing } \\
\text { the use of } \\
\text { both internal } \\
\text { and external } \\
\text { supporters. } \\
\text { Offside rule not } \\
\text { included }\end{array}$ & $\begin{array}{l}\text { Pass } \\
\text { Dribbling } \\
\text { Collective success } \\
\text { Game pause }\end{array}$ & $\begin{array}{l}4 \text { vs. } 4 \text { formats (with and } \\
\text { without GK) increased the } \\
\text { number of dribbling in } \\
\text { comparison with formats } \\
\text { with neutral players. } 4 \text { vs. } 4 \\
\text { formats without GK (with } \\
\text { and without neutral players) } \\
\text { meaningfully increased the } \\
\text { collective success compared } \\
\text { with formats with GK }\end{array}$ & 86.7 \\
\hline [26] & $\begin{array}{c}27 \text { soccer } \\
\text { players, } \\
\text { under-12 (age } \\
\text { not described) }\end{array}$ & $\begin{array}{c}\text { Analyse } \\
\text { the impact } \\
\text { of changes } \\
\text { in format } \\
\text { on technical } \\
\text { actions }\end{array}$ & $\begin{array}{c}8 \text { vs. } 8 \\
11 \text { vs. } 11\end{array}$ & $\begin{array}{l}62 \times 51 \mathrm{~m} \\
80 \times 54 \mathrm{~m}\end{array}$ & $\begin{array}{l}198 \mathrm{~m}^{2} \\
196 \mathrm{~m}^{2}\end{array}$ & $1 \times 25^{\prime}$ & $\begin{array}{l}\text { No shoot directly } \\
\text { at kick-off; could } \\
\text { not pass the ball } \\
\text { over the halfway } \\
\text { line at goal kick; } \\
\text { GK could not kick } \\
\text { over the half-way }\end{array}$ & $\begin{array}{l}\text { Ball touches } \\
\text { Passes } \\
\text { Passes in attacking area } \\
\text { Passing in attacking } \\
1 / 3 \text { area } \\
\text { Passes in defence area } \\
\text { One-touch pass } \\
\text { Forward passes } \\
\text { Shot, mid-, and long- } \\
\text { distance passes } \\
\text { Shots } \\
\text { Goals } \\
\text { Duels } \\
\text { Cutbacks } \\
\text { Crosses } \\
\text { Dribbles }\end{array}$ & $\begin{array}{l}\text { The smaller format meaning- } \\
\text { fully increased the frequencies } \\
\text { of ball touches, all passes, } \\
\text { passes in different areas and } \\
\text { the success of those passes, } \\
\text { short and mid-distance passes, } \\
\text { goal shots, shots in penalty } \\
\text { area, and successful dribbles }\end{array}$ & 86.7 \\
\hline
\end{tabular}

yo - years old, CV - coefficient of variation, GK - goalkeeper, SGG - small-sided game

* excluding GK, \# the relative area per player excluded the 2 external neutral players 


\section{HUMAN MOVEMENT}

F. Clemente, H. Sarmento, Small-sided games and technical actions: a review

Table 2. Effects of manipulation of pitch size, relative area per player, pitch restrictions and configurations on technical actions

\begin{tabular}{|c|c|c|c|c|c|c|c|c|c|c|}
\hline 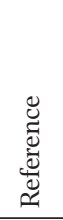 & 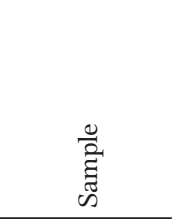 & 妾 & 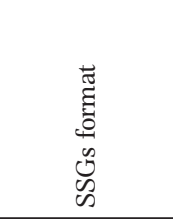 & 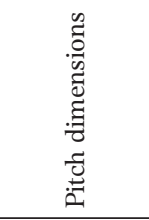 & 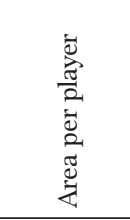 & 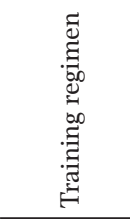 & $\frac{\mathscr{U}}{\Xi}$ & 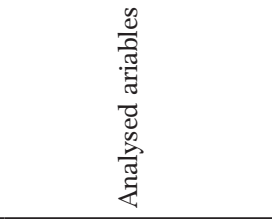 & 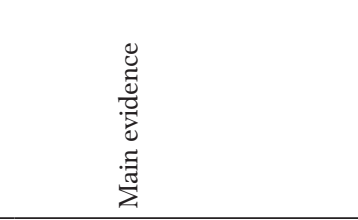 & 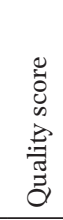 \\
\hline [29] & $\begin{array}{l}8 \text { soccer } \\
\text { players, } \\
18 \pm 1 \text { yo }\end{array}$ & $\begin{array}{l}\text { Analyse } \\
\text { the impact } \\
\text { of changes } \\
\text { in pitch size } \\
\text { on technical } \\
\text { actions }\end{array}$ & 5 vs. $5+\mathrm{GK}$ & $\begin{array}{l}30 \times 20 \mathrm{~m} \\
40 \times 30 \mathrm{~m} \\
50 \times 40 \mathrm{~m}\end{array}$ & $\begin{array}{r}60 \mathrm{~m}^{2} \\
120 \mathrm{~m}^{2} \\
200 \mathrm{~m}^{2}\end{array}$ & $\begin{array}{l}4 \times 4^{\prime} / 2^{\prime} \\
\text { active } \\
\text { recovery }\end{array}$ & $\begin{array}{c}\text { No specific } \\
\text { rules }\end{array}$ & $\begin{array}{l}\text { Pass } \\
\text { Received } \\
\text { Turn } \\
\text { Dribble } \\
\text { Header } \\
\text { Tackle } \\
\text { Interception } \\
\text { Shot } \\
\text { Target pass }\end{array}$ & $\begin{array}{l}\text { Significant increases } \\
\text { of tackles and shots occurred } \\
\text { on smaller pitch dimensions. } \\
\text { Pitch size does not seem } \\
\text { to significantly change } \\
\text { the technical actions }\end{array}$ & 86.7 \\
\hline [30] & $\begin{array}{c}10 \text { soccer } \\
\text { players, } \\
15.5 \pm 0.5 \text { yo }\end{array}$ & $\begin{array}{l}\text { Analyse } \\
\text { the impact } \\
\text { of changes } \\
\text { in pitch size } \\
\text { on technical } \\
\text { actions }\end{array}$ & 5 vs. $5+\mathrm{GK}$ & $\begin{array}{l}32 \times 23 \mathrm{~m} \\
50 \times 35 \mathrm{~m} \\
62 \times 44 \mathrm{~m}\end{array}$ & $\begin{array}{r}74 \mathrm{~m}^{2} \\
175 \mathrm{~m}^{2} \\
273 \mathrm{~m}^{2}\end{array}$ & $1 \times 8^{\prime}$ & $\begin{array}{l}\text { No offside } \\
\text { rule }\end{array}$ & $\begin{array}{l}\text { Tackle } \\
\text { Interception } \\
\text { Control } \\
\text { Control and dribble } \\
\text { Control, dribble, } \\
\text { and pass } \\
\text { Control and pass } \\
\text { Control and shoot } \\
\text { Header } \\
\text { First-touch pass } \\
\text { Clearance } \\
\text { Putting ball in play }\end{array}$ & $\begin{array}{l}\text { Smaller pitch dimension } \\
\text { presented significant } \\
\text { increases of clearance and } \\
\text { putting ball in play compared } \\
\text { with the remaining dimen- } \\
\text { sions. Smaller format had } \\
\text { significant increases } \\
\text { of interception, control, } \\
\text { and dribble compared with } \\
\text { the larger pitch. The effective } \\
\text { playing time was higher in } \\
\text { the largest pitch }\end{array}$ & 86.7 \\
\hline [31] & $\begin{array}{l}10 \text { soccer } \\
\text { players, } \\
31.7 \pm 7.6 \text { yo } \\
\text { (recreational } \\
\text { players) }\end{array}$ & $\begin{array}{l}\text { Analyse } \\
\text { the impact } \\
\text { of different } \\
\text { pitch sizes } \\
\text { and formats } \\
\text { on technical } \\
\text { actions }\end{array}$ & $\begin{array}{l}5 \text { vs. } 5+\mathrm{GK} \\
5 \text { vs. } 5+\mathrm{GK} \\
7 \text { vs. } 7+\mathrm{GK} \\
7 \text { vs. } 7+\mathrm{GK}\end{array}$ & $\begin{array}{l}44 \times 23 \mathrm{~m} \\
57 \times 30 \mathrm{~m} \\
44 \times 23 \mathrm{~m} \\
57 \times 30 \mathrm{~m}\end{array}$ & $\begin{array}{c}101 \text { and } \\
171 \mathrm{~m}^{2} \\
\text { for } 5 \text { vs. } 5 \\
72 \text { and } \\
122 \mathrm{~m}^{2} \\
\text { for } 7 \text { vs. } 7\end{array}$ & $1 \times 40^{\prime}$ & $\begin{array}{c}\text { There were } \\
\text { no throw-ins } \\
\text { The matches } \\
\text { occurred } \\
\text { in outdoor and } \\
\text { sand carpet }\end{array}$ & $\begin{array}{l}\text { Ball possessions } \\
\text { Dribbling } \\
\text { Successful pass } \\
\text { Unsuccessful pass } \\
\text { Tackle } \\
\text { Shot }\end{array}$ & $\begin{array}{l}\text { Ball possessions were } \\
\text { significantly greater on the } \\
\text { smaller pitch. Unsuccessful } \\
\text { passes were also significantly } \\
\text { greater on the smaller pitch }\end{array}$ & 86.7 \\
\hline [32] & $\begin{array}{c}32 \text { soccer } \\
\text { players, } \\
12.0 \pm 0.4 \text { yo }\end{array}$ & $\begin{array}{c}\text { Analyse } \\
\text { the impact } \\
\text { of different } \\
\text { pitch } \\
\text { dimensions } \\
\text { on technical } \\
\text { actions }\end{array}$ & $\begin{array}{c}7 \text { vs. } 7+\mathrm{GK} \\
7 \text { vs. } 7+\mathrm{GK} \\
11 \text { vs. } 11 \\
\text { (regular) }\end{array}$ & $\begin{array}{l}68 \times 47 \mathrm{~m} \\
75 \times 47 \mathrm{~m} \\
75 \times 47 \mathrm{~m}\end{array}$ & $\begin{array}{l}228 \mathrm{~m}^{2} \\
252 \mathrm{~m}^{2} \\
176 \mathrm{~m}^{2}\end{array}$ & $1 \times 30^{\prime}$ & Regular rules & $\begin{array}{l}\text { Blocked balls } \\
\text { Back passes } \\
\text { Side passes } \\
\text { Forward passes } \\
\text { Short-distance passes } \\
\text { Mid-distance passes } \\
\text { Long-distance passes } \\
\text { Continuous passes } \\
\text { All passes }\end{array}$ & $\begin{array}{l}\text { The } 7 \text { vs. } 7+\text { GK format } \\
\text { played in the bigger pitch } \\
\text { dimension significantly } \\
\text { increased the number } \\
\text { of forward passes and } \\
\text { all passes }\end{array}$ & 93.3 \\
\hline [33] & $\begin{array}{c}33 \text { soccer } \\
\text { players, } \\
10.0 \pm 0.5 \text { yo } \\
(n=17), 13.2 \\
\pm 0.3 \text { yo }(n \\
=16)\end{array}$ & $\begin{array}{l}\text { Analyse } \\
\text { the impact } \\
\text { of different } \\
\text { relative space } \\
\text { per player } \\
\text { on technical } \\
\text { actions }\end{array}$ & $\begin{array}{l}3 \text { vs. } 3 \\
3 \text { vs. } 3 \\
4 \text { vs. } 4 \\
4 \text { vs. } 4 \\
5 \text { vs. } 5 \\
5 \text { vs. } 5\end{array}$ & $\begin{array}{l}20 \times 20 \mathrm{~m} \\
30 \times 30 \mathrm{~m} \\
20 \times 20 \mathrm{~m} \\
30 \times 30 \mathrm{~m} \\
20 \times 20 \mathrm{~m} \\
30 \times 30 \mathrm{~m}\end{array}$ & $\begin{array}{r}67 \mathrm{~m}^{2} \\
150 \mathrm{~m}^{2} \\
\\
50 \mathrm{~m}^{2} \\
113 \mathrm{~m}^{2} \\
\\
40 \mathrm{~m}^{2} \\
90 \mathrm{~m}^{2}\end{array}$ & $\begin{array}{l}3 \times 4 ' / 3, \\
\text { active } \\
\text { recovery }\end{array}$ & $\begin{array}{c}\text { No GK. } \\
\text { Goals were } \\
\text { only valid } \\
\text { when all the team } \\
\text { were within } \\
\text { the opponent's } \\
\text { half }\end{array}$ & $\begin{array}{l}\text { Involvements with } \\
\text { the ball } \\
\text { Crosses } \\
\text { Headers } \\
\text { Tackles } \\
\text { Shots on goal } \\
\text { Dribbles } \\
\text { Passes } \\
\text { Target passes }\end{array}$ & $\begin{array}{l}\text { The older group performed } \\
\text { more dribbles, crosses, and } \\
\text { tackles in the case of smaller } \\
\text { relative space. In greater } \\
\text { relative space, greater values } \\
\text { of involvements with the ball } \\
\text { were found. A scattered } \\
\text { distribution of technical } \\
\text { actions among different } \\
\text { relative spaces was observed } \\
\text { among younger players }\end{array}$ & 86.7 \\
\hline
\end{tabular}




\begin{tabular}{|c|c|c|c|c|c|c|c|c|c|c|}
\hline [34] & $\begin{array}{c}13 \text { soccer } \\
\text { players, } \\
16.6 \pm 0.9 \text { yo }\end{array}$ & $\begin{array}{c}\text { Analyse } \\
\text { the impact } \\
\text { of different } \\
\text { pitch } \\
\text { dimensions } \\
\text { on technical } \\
\text { actions made } \\
\text { by GK }\end{array}$ & 5 vs. $5+$ GK & $\begin{array}{l}32 \times 23 \mathrm{~m} \\
50 \times 35 \mathrm{~m} \\
62 \times 44 \mathrm{~m}\end{array}$ & $\begin{array}{r}74 \mathrm{~m}^{2} \\
175 \mathrm{~m}^{2} \\
273 \mathrm{~m}^{2}\end{array}$ & $3 \times 8^{\prime} / 5^{\prime}$ & $\begin{array}{l}\text { There were } \\
\text { no corners }\end{array}$ & $\begin{array}{l}\text { Save } \\
\text { Parry } \\
\text { Clear-out } \\
\text { Deflection } \\
\text { Open palm } \\
\text { technique } \\
\text { Fly } \\
\text { Screen } \\
\text { Control with the foot } \\
\text { Clear-out by the defence } \\
\text { 1-on-1 } \\
\text { Goal kick } \\
\text { Direct and indirect } \\
\text { free kick } \\
\text { Pass by hand and foot }\end{array}$ & $\begin{array}{l}\text { The larger pitch increased the } \\
\text { frequencies of duels and } \\
\text { blocks. The smaller pitch } \\
\text { increased the number } \\
\text { of passes performed } \\
\text { with both hand and foot }\end{array}$ & 86.7 \\
\hline [35] & $\begin{array}{c}148 \text { soccer } \\
\text { players, } \\
12.5 \pm 0.5 \text { yo } \\
(n=36), \\
14.4 \pm 0.5 \text { yo } \\
(n=43), \\
16.6 \pm 3.2 \text { yo } \\
(n=28), \\
17.9 \pm 1.0 \text { yo } \\
(n=43)\end{array}$ & $\begin{array}{c}\text { Analyse } \\
\text { the impact } \\
\text { of different } \\
\text { pitch } \\
\text { dimensions } \\
\text { on technical } \\
\text { actions }\end{array}$ & $\begin{array}{l}4 \text { vs. } 4+\mathrm{GK} \\
4 \text { vs. } 4+\mathrm{GK}\end{array}$ & $\begin{array}{l}40 \times 30 \mathrm{~m} \\
68 \times 47 \mathrm{~m}\end{array}$ & $\begin{array}{l}120 \mathrm{~m}^{2} \\
320 \mathrm{~m}^{2}\end{array}$ & $1 \times 4^{\prime}$ & $\begin{array}{l}\text { Offside rule was } \\
\text { not applied in } \\
\text { the smaller pitch } \\
\text { dimension }\end{array}$ & $\begin{array}{l}\text { Transitions } \\
\text { Ball possessions } \\
\text { Set pieces } \\
\text { Goals/shots }\end{array}$ & $\begin{array}{l}\text { The bigger pitch led } \\
\text { to longer periods of ball } \\
\text { possession and less } \\
\text { transitions }\end{array}$ & 86.7 \\
\hline [36] & $\begin{array}{c}20 \text { soccer } \\
\text { players, } \\
14.1 \pm 0.5 \text { yo }\end{array}$ & $\begin{array}{l}\text { Analyse } \\
\text { the effects } \\
\text { of different } \\
\text { pitch formats } \\
\text { (manipulating } \\
\text { the length } \\
\text { and width) } \\
\text { on technical } \\
\text { actions }\end{array}$ & 4 vs. $4+$ GK & $\begin{array}{l}40 \times 30 \mathrm{~m} \\
30 \times 40 \mathrm{~m}\end{array}$ & $\begin{array}{l}150 \mathrm{~m}^{2} \\
150 \mathrm{~m}^{2}\end{array}$ & $\begin{array}{c}3 \times 6{ }^{\prime} / 3^{\prime} \\
\text { rest }\end{array}$ & $\begin{array}{c}\text { Offside was } \\
\text { not applied }\end{array}$ & $\begin{array}{l}\text { Passes } \\
\text { Lost balls } \\
\text { Dribbles } \\
\text { Shoots } \\
\text { Goals }\end{array}$ & $\begin{array}{l}\text { The } 30 \times 40 \mathrm{~m} \text { led to } \\
\text { meaningful increases in the } \\
\text { number of shots per player. } \\
\text { The } 40 \times 30 \mathrm{~m} \text { meaningfully } \\
\text { increased the completed } \\
\text { passes per player }\end{array}$ & 86.7 \\
\hline [37] & $\begin{array}{c}10 \text { soccer } \\
\text { players, } \\
13.7 \pm 0.5 \text { yo }\end{array}$ & $\begin{array}{c}\text { Analyse } \\
\text { the effects } \\
\text { of different } \\
\text { external } \\
\text { markings } \\
\text { of the pitch } \\
\text { on technical } \\
\text { actions }\end{array}$ & 5 vs. $5+$ GK & $30 \times 25 \mathrm{~m}$ & $75 \mathrm{~m}^{2}$ & $\begin{array}{c}3 \times 6{ }^{\prime} / 3^{\prime} \\
\text { rest }\end{array}$ & $\begin{array}{l}\text { Three different } \\
\text { external markings } \\
\text { were used: (1) } \\
\text { complete lines, (2) } \\
\text { dashed lines, and } \\
\text { (3) painted marker } \\
\text { in the corners }\end{array}$ & $\begin{array}{l}\text { Passes } \\
\text { Dribbles } \\
\text { Shots on target } \\
\text { Goals }\end{array}$ & $\begin{array}{l}\text { Compared with corners, the } \\
\text { lines presented the number } \\
\text { of offensive technical } \\
\text { indicators. Lines revealed } \\
\text { a lower number of passes }\end{array}$ & 93.3 \\
\hline
\end{tabular}

yo - years old, GK - goalkeeper, SGG - small-sided game 
Table 3. Effects of different task conditions on technical actions

\begin{tabular}{|c|c|c|c|c|c|c|c|c|c|c|}
\hline 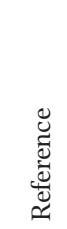 & 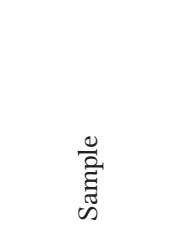 & 妾 & $\begin{array}{l}\text { है } \\
\text { है } \\
\infty \\
\infty \\
\infty \\
\infty\end{array}$ & 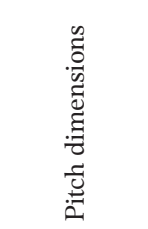 & 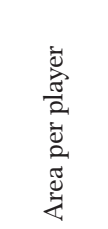 & 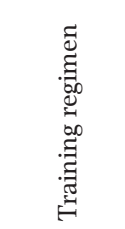 & $\frac{\mathscr{Q}}{\vec{Z}}$ & 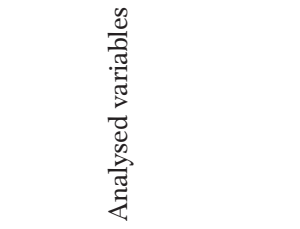 & 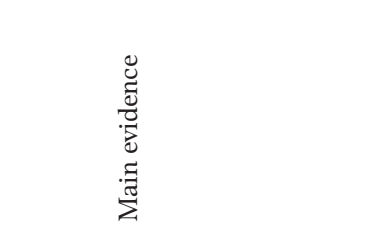 & 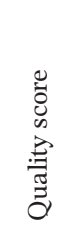 \\
\hline [39] & $\begin{array}{l}20 \text { soccer } \\
\text { players, } \\
27.4 \pm 1.5 \\
\text { yo (national } \\
\text { team) }\end{array}$ & $\begin{array}{l}\text { Analyse } \\
\text { the impact } \\
\text { of different } \\
\text { ball-touch } \\
\text { limitations } \\
\text { ( } 1 \text { touch, } \\
2 \text { touches, } \\
\text { and free play) } \\
\text { on technical } \\
\text { actions }\end{array}$ & 4 vs. 4 & $30 \times 20 \mathrm{~m}$ & $75 \mathrm{~m}^{2}$ & $\begin{array}{c}4 \times 4^{\prime} / 3^{\prime} \\
\text { passive } \\
\text { recovery }\end{array}$ & $\begin{array}{l}\text { Keep the ball as } \\
\text { long as possible } \\
\text { for a given team } \\
\text { using } 4 \text { support } \\
\text { players outside } \\
\text { of the pitch }\end{array}$ & $\begin{array}{l}\text { Number of duels } \\
\text { Percentage of successful } \\
\text { passes } \\
\text { Number of balls lost } \\
\text { Total number of ball } \\
\text { possessions }\end{array}$ & $\begin{array}{l}\text { Free-play rule increased the } \\
\text { number of duels in each bout } \\
\text { and affected less the technical } \\
\text { actions (successful passes } \\
\text { and number of ball losses) } \\
\text { from bout } 1 \text { to last compared } \\
\text { with the } 1 \text { - and } 2 \text {-ball touch } \\
\text { limitations. The } 1 \text {-ball touch } \\
\text { limitation increased the total } \\
\text { number of ball possessions } \\
\text { but significantly decreased } \\
\text { the \% of successful passes } \\
\text { compared with the remaining } \\
\text { conditions }\end{array}$ & 86.7 \\
\hline [38] & $\begin{array}{l}20 \text { soccer } \\
\text { players, } \\
27.4 \pm 1.5 \\
\text { yo (national } \\
\text { team) }\end{array}$ & $\begin{array}{c}\text { Analyse } \\
\text { the impact } \\
\text { of different } \\
\text { ball-touch } \\
\text { limitations } \\
\text { (1 touch, } \\
2 \text { touches, } \\
\text { and free play) } \\
\text { on technical } \\
\text { actions }\end{array}$ & $\begin{array}{l}2 \text { vs. } 2 \\
3 \text { vs. } 3 \\
4 \text { vs. } 4\end{array}$ & $\begin{array}{l}20 \times 15 \mathrm{~m} \\
25 \times 18 \mathrm{~m} \\
30 \times 20 \mathrm{~m}\end{array}$ & $\begin{array}{l}75 \mathrm{~m}^{2} \\
75 \mathrm{~m}^{2} \\
75 \mathrm{~m}^{2}\end{array}$ & $\begin{array}{c}4 \times 2 ' / 2 \text { ' } \\
\text { passive } \\
\text { recovery } \\
4 \times 3 \text { ' } / 3 \text { ' } \\
\text { passive } \\
\text { recovery } \\
4 \times 4 \text { ' } / 4 \text { ' } \\
\text { passive } \\
\text { recovery }\end{array}$ & $\begin{array}{l}\text { Keep the ball as } \\
\text { long as possible } \\
\text { for a given team } \\
\text { using } 4 \text { support } \\
\text { players outside } \\
\text { of the pitch }\end{array}$ & $\begin{array}{l}\text { Number of duels } \\
\text { Duels per minute of play } \\
\text { Percentage of successful } \\
\text { passes } \\
\text { Number of balls lost } \\
\text { Balls lost per minute } \\
\text { of play } \\
\text { Total number of ball } \\
\text { possessions }\end{array}$ & $\begin{array}{l}\text { The free-play condition } \\
\text { contributed to the largest } \\
\text { number of duels per minute } \\
\text { in the } 4 \text { vs. } 4 \text {; however, during } \\
\text { the } 2 \text { vs. } 2 \text {, the largest number } \\
\text { occurred in the } 2 \text {-touch } \\
\text { condition. The } 1 \text {-touch } \\
\text { limitation led to the smallest } \% \\
\text { of successful passes and to } \\
\text { the greatest number of balls } \\
\text { lost per minute and total } \\
\text { number of possessions }\end{array}$ & 86.7 \\
\hline [43] & $\begin{array}{c}16 \text { soccer } \\
\text { players, } \\
15.8 \pm 0.5 \text { yo }\end{array}$ & $\begin{array}{c}\text { Analyse } \\
\text { the effects } \\
\text { of different } \\
\text { task conditions } \\
\text { (playing } \\
\text { only offense, } \\
\text { playing only } \\
\text { defence, both) } \\
\text { on technical } \\
\text { actions }\end{array}$ & $\begin{array}{l}3 \text { vs. } 3+\mathrm{GK} \\
4 \text { vs. } 4+\mathrm{GK} \\
\end{array}$ & $\begin{array}{l}30 \times 20 \mathrm{~m} \\
40 \times 20 \mathrm{~m}\end{array}$ & $\begin{array}{l}100 \mathrm{~m}^{2} \\
100 \mathrm{~m}^{2}\end{array}$ & $\begin{array}{c}4 \times 4^{\prime} / 2 \text { ' } \\
\text { active } \\
\text { recovery }\end{array}$ & Not mentioned & $\begin{array}{l}\text { Pass } \\
\text { Receive } \\
\text { Dribble } \\
\text { Shot } \\
\text { Tackle } \\
\text { Interception }\end{array}$ & $\begin{array}{l}\text { No meaningful differences } \\
\text { in technical actions between } \\
\text { formats or task conditions. } \\
\text { There was interaction in passes; } \\
\text { higher pass effectiveness in the } \\
4 \text { vs. } 4 \text { (free - both situations) } \\
\text { compared with } 3 \text { vs. } 3 \text { (free - } \\
\text { both situations) }\end{array}$ & 86.7 \\
\hline [40] & $\begin{array}{c}10 \text { soccer } \\
\text { players, } \\
26.4 \pm 5.3 \text { yo } \\
\text { (amateurs) }\end{array}$ & $\begin{array}{c}\text { Analyse } \\
\text { the effects } \\
\text { of } 3 \text { different } \\
\text { scoring } \\
\text { methods } \\
\text { (line to cross, } \\
2 \text { small goals, } \\
\text { and } 1 \text { central } \\
\text { small goal) } \\
\text { on technical } \\
\text { actions }\end{array}$ & $\begin{array}{l}2 \text { vs. } 2+2 \\
3 \text { vs. } 3+2 \\
4 \text { vs. } 4+2\end{array}$ & $\begin{array}{l}19 \times 19 \mathrm{~m} \\
23 \times 23 \mathrm{~m} \\
27 \times 27 \mathrm{~m}\end{array}$ & $\begin{array}{l}90 \mathrm{~m}^{2} \\
90 \mathrm{~m}^{2} \\
90 \mathrm{~m}^{2}\end{array}$ & $\begin{array}{c}3 \times 5, / 3, \\
\text { rest }\end{array}$ & $\begin{array}{l}\text { The } 2 \text { neutral } \\
\text { players provided } \\
\text { numerical } \\
\text { advantage to the } \\
\text { team with ball } \\
\text { possession. In } \\
\text { the line to cross } \\
\text { condition, the } \\
\text { point was earned } \\
\text { every time that a } \\
\text { player received } \\
\text { the ball behind } \\
\text { the line from his } \\
\text { teammate }\end{array}$ & $\begin{array}{l}\text { Conquered balls } \\
\text { Received balls } \\
\text { Lost balls and neutral } \\
\text { balls } \\
\text { Pass } \\
\text { Successful shot on goal } \\
\text { The technical actions } \\
\text { were then converted } \\
\text { into the following items: } \\
\text { volume of play, attacks } \\
\text { with ball, efficiency } \\
\text { index, and performance } \\
\text { score }\end{array}$ & $\begin{array}{l}\text { Significantly greater efficiency } \\
\text { index, performance score, } \\
\text { and attacks with ball were } \\
\text { found in line to cross condition. } \\
\text { Comparisons between formats } \\
\text { revealed significantly greater } \\
\text { values of volume of play and } \\
\text { efficiency index in the smaller } \\
\text { format }(2 \text { vs. } 2+2)\end{array}$ & 93.3 \\
\hline$[41]$ & $\begin{array}{c}24 \text { soccer } \\
\text { players, } \\
19.1 \pm 1.2 \text { yo }\end{array}$ & $\begin{array}{l}\text { Analyse } \\
\text { the effects } \\
\text { of different } \\
\text { scoring } \\
\text { methods } \\
\text { (small goals, } \\
\text { GKs, floaters) } \\
\text { on technical } \\
\text { actions }\end{array}$ & $\begin{array}{c}7 \text { vs. } 7+\mathrm{GK} \\
7 \text { vs. } 7 \\
7 \text { vs. } 7+\mathrm{GK} \\
+2 \text { outside } \\
\text { neutral players }\end{array}$ & $40 \times 25 \mathrm{~m}$ & $71 \mathrm{~m}^{2}$ & $1 \times 6$ & $\begin{array}{c}\text { The } 7 \text { vs. } 7 \\
\text { without GK } \\
\text { used small goals. } \\
\text { No offside rule }\end{array}$ & $\begin{array}{l}\text { Percentage of total } \\
\text { possession } \\
\text { Offensive sequences } \\
\text { (duration) }\end{array}$ & $\begin{array}{l}\text { Formats using small goals } \\
\text { and neutral players had greater } \\
\text { possession duration compared } \\
\text { with the format with only GK }\end{array}$ & 93.3 \\
\hline
\end{tabular}




\begin{tabular}{|c|c|c|c|c|c|c|c|c|c|c|}
\hline [42] & $\begin{array}{c}8 \text { soccer } \\
\text { players, } \\
12.1 \pm 0.5 \text { yo }\end{array}$ & $\begin{array}{l}\text { Analyse } \\
\text { the effects } \\
\text { of different } \\
\text { scoring } \\
\text { methods } \\
\text { (2-goal game, } \\
\text { 4-goal game, } \\
\text { 2-goal game } \\
\text { with goal- } \\
\text { posts, 4-goal } \\
\text { game with } \\
1 \text { goal } \\
\text { positioned } \\
\text { infield each } \\
\text { corner) on } \\
\text { technical } \\
\text { actions }\end{array}$ & 4 vs. 4 & $\begin{array}{l}45.72 \times \\
36.58 \mathrm{~m}\end{array}$ & $209 \mathrm{~m}^{2}$ & $2 \times 5^{\prime} / 3^{\prime}$ & $\begin{array}{l}\text { Scoring methods } \\
\text { were: (1) 2-goal } \\
\text { game, (2) 4-goal } \\
\text { game, (3) 2-goal } \\
\text { game with goal- } \\
\text { posts, (4) 4-goal } \\
\text { game with } 1 \text { goal } \\
\text { positioned infield } \\
\text { each corner. There } \\
\text { were no throw-ins } \\
\text { during the games }\end{array}$ & $\begin{array}{l}\text { Pass forwards } \\
\text { Pass sideways } \\
\text { Pass backwards } \\
\text { Successful pass } \\
\text { Unsuccessful pass } \\
\text { Penetrating pass } \\
\text { Turning } \\
\text { Dribbling } \\
\text { Shot } \\
\text { Goal } \\
\text { Overlap } \\
\text { One-two } \\
1 \text { vs. 1 (duels) }\end{array}$ & $\begin{array}{l}\text { Higher frequencies of } \\
\text { successful pass forwards and } \\
\text { backwards and penetrating } \\
\text { pass occurred in the 2-goal } \\
\text { game condition. Successful } \\
\text { pass sideways occurred more } \\
\text { often in the 4-goal game. More } \\
\text { turnings were found in the } \\
\text { 4-goal condition. Shots and } \\
\text { goals were more frequent in } \\
\text { the condition of } 4 \text {-goal game } \\
\text { with } 1 \text { goal positioned infield } \\
\text { each corner }\end{array}$ & 93.3 \\
\hline [45] & $\begin{array}{c}8 \text { soccer } \\
\text { players, } \\
20.1 \pm 1.0 \text { yo } \\
\text { (amateurs) }\end{array}$ & $\begin{array}{l}\text { Analyse } \\
\text { the effects } \\
\text { of the type } \\
\text { of floor (sand, } \\
\text { artificial turf) } \\
\text { on technical } \\
\text { actions }\end{array}$ & 4 vs. $4+\mathrm{GK}$ & $37 \times 28 \mathrm{~m}$ & $130 \mathrm{~m}^{2}$ & $\begin{array}{c}3 \times 6^{\prime} / 3^{\prime} \\
\text { rest }\end{array}$ & No offside rule & $\begin{array}{l}\text { Involvements } \\
\text { with the ball } \\
\text { Passes or crosses } \\
\text { Headers } \\
\text { Chest trap } \\
\text { Tackles } \\
\text { Dribbling } \\
\text { Shots } \\
\text { Goals }\end{array}$ & $\begin{array}{l}\text { More succeeded actions were } \\
\text { achieved in artificial turf in } \\
\text { comparison with sand. Actions } \\
\text { requiring lifting the ball } \\
\text { were higher on sand than on } \\
\text { artificial sand }\end{array}$ & 86.7 \\
\hline [44] & $\begin{array}{c}16 \text { soccer } \\
\text { players, } \\
23.9 \pm 5.4 \text { yo } \\
\text { (semi- } \\
\text { professional) }\end{array}$ & $\begin{array}{l}\text { Analyse } \\
\text { the effects } \\
\text { of coach's } \\
\text { instruction } \\
\text { on technical } \\
\text { actions }\end{array}$ & 7 vs. $7+\mathrm{GK}$ & $62 \times 50 \mathrm{~m}$ & $221 \mathrm{~m}^{2}$ & $1 \times 5$ & $\begin{array}{c}\text { The teams } \\
\text { received no } \\
\text { instructions and } \\
\text { instructions about } \\
\text { defensive and } \\
\text { offensive strategy. } \\
\text { Regular rules were } \\
\text { followed }\end{array}$ & $\begin{array}{l}\text { Ball recoveries } \\
\text { Number of passes } \\
\text { per ball possession }\end{array}$ & $\begin{array}{l}\text { Defensive instruction } \\
\text { increased recovered balls and } \\
\text { decreased the space occupied. } \\
\text { Offensive instructions resulted } \\
\text { in greater number of passes } \\
\text { and increased the space } \\
\text { occupied compared with } \\
\text { control group (no instructions) }\end{array}$ & 93.3 \\
\hline [46] & $\begin{aligned} & 20 \text { soccer } \\
& \text { players, } \\
& 13.5 \pm 1.2 \text { yo } \\
&(n=10) \\
& 16.3 \pm 0.5 \text { yo } \\
&(n=10)\end{aligned}$ & $\begin{array}{l}\text { Analyse } \\
\text { the effects } \\
\text { of changing } \\
\text { rules and } \\
\text { configurations } \\
\text { on technical } \\
\text { actions }\end{array}$ & $\begin{array}{l}3 \text { vs. } 3+\mathrm{GK} \\
4 \text { vs. } 4+\mathrm{GK}\end{array}$ & $\begin{array}{c}36 \times 27 \mathrm{~m} \\
47.72 \times \\
29.54 \mathrm{~m}\end{array}$ & $\begin{array}{l}162 \mathrm{~m}^{2} \\
176 \mathrm{~m}^{2}\end{array}$ & $1 \times 10^{\prime}$ & $\begin{array}{l}\text { Three } \\
\text { representative } \\
\text { SSGs, } 3 \\
\text { maintaining ball } \\
\text { possessions, and } \\
3 \text { progressions to } \\
\text { target games were } \\
\text { implemented }\end{array}$ & $\begin{array}{l}\text { Ball possessions } \\
\text { Players involved } \\
\text { Ball touches } \\
\text { Passes } \\
\text { Shots }\end{array}$ & $\begin{array}{l}\text { Smaller SSGs configurations } \\
\text { increased the difficulties of } \\
\text { keeping ball possession in } \\
\text { younger players. The } 3 \text { vs. } 3 \\
\text { format promoted better } \\
\text { offensive efficacy in progression } \\
\text { games. The condition of main- } \\
\text { taining ball possession contri- } \\
\text { buted to longer passing } \\
\text { sequences and more players } \\
\text { involved }\end{array}$ & 86.7 \\
\hline
\end{tabular}

yo - years old, GK - goalkeeper, SGG - small-sided game 


\section{HUMAN MOVEMENT}

F. Clemente, H. Sarmento, Small-sided games and technical actions: a review

Table 4. Effects of different age groups, experience, and technical level

\begin{tabular}{|c|c|c|c|c|c|c|c|c|c|c|}
\hline 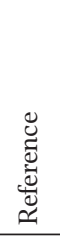 & 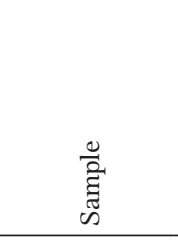 & 寻 & 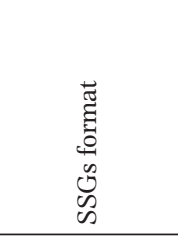 & 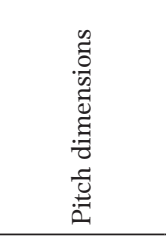 & 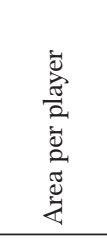 & 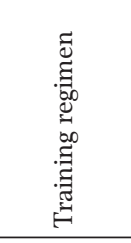 & $\frac{0}{3}$ & 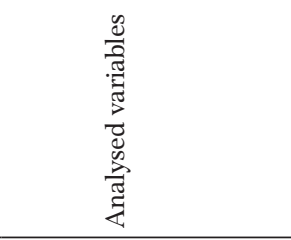 & 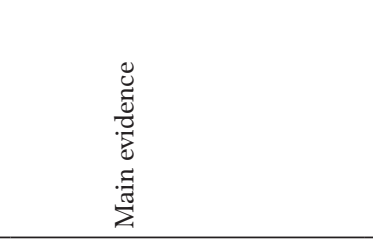 & 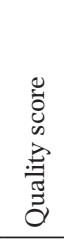 \\
\hline [47] & $\begin{array}{c}28 \text { soccer } \\
\text { players, } \\
12.8 \pm 0.6 \text { yo } \\
(n=14 \text { non- } \\
\text { experienced }), \\
12.9 \pm 0.6 \text { yo } \\
(n=14 \\
\text { experienced })\end{array}$ & $\begin{array}{c}\text { Analyse } \\
\text { the interaction } \\
\text { between } \\
\text { experience } \\
\text { of players } \\
\text { and play format } \\
\text { on the technical } \\
\text { actions }\end{array}$ & $\begin{array}{l}3 \text { vs. } 3+\mathrm{GK} \\
6 \text { vs. } 6+\mathrm{GK}\end{array}$ & $\begin{array}{c}46 \times 31 \mathrm{~m} \\
62 \times 40.4 \mathrm{~m}\end{array}$ & $\begin{array}{l}238 \mathrm{~m}^{2} \\
209 \mathrm{~m}^{2}\end{array}$ & $\begin{array}{c}2 \times 5^{\prime} / 1 \\
\text { rest }\end{array}$ & $\begin{array}{l}\text { Offside rule } \\
\text { was not } \\
\text { applied }\end{array}$ & $\begin{array}{l}\text { Duration of ball } \\
\text { possession } \\
\text { Players involved } \\
\text { Ball touches } \\
\text { Passes } \\
\text { Players involved/ } \\
\text { duration } \\
\text { Ball touches/duration } \\
\text { Passes/duration } \\
\text { Ball touches/players } \\
\text { involved } \\
\text { Passes/players involved } \\
\text { Passes/ball touches } \\
\text { Shots } \\
\text { Goal/shots } \\
\text { Offensive sequences }\end{array}$ & $\begin{array}{l}\text { Experienced players made } \\
\text { significantly longer offensive } \\
\text { sequences, with a great number } \\
\text { of players involved that also } \\
\text { executed more touches on the } \\
\text { ball and more passing actions. } \\
\text { Non-experienced players tended } \\
\text { to build attacks based on indivi- } \\
\text { dual actions; experienced } \\
\text { players opted for ball posses- } \\
\text { sion style. Finalization was not } \\
\text { different between experienced } \\
\text { and non-experienced players } \\
\text { in any format }\end{array}$ & 86.7 \\
\hline [48] & $\begin{array}{c}48 \text { soccer } \\
\text { players, } \\
13.1 \pm 1.5 \text { yo } \\
(n=24), \\
16.9 \pm 0.1 \text { yo } \\
(n=24)\end{array}$ & $\begin{array}{l}\text { Analyse } \\
\text { the effects } \\
\text { of age } \\
\text { and tactical } \\
\text { skills on } \\
\text { technical actions }\end{array}$ & $\begin{array}{c}3 \text { vs. } 3+3+ \\
\text { GK } \\
4 \text { vs. } 4+\text { GK }\end{array}$ & $\begin{array}{l}47.72 \times \\
29.54 \mathrm{~m} \\
47.72 \times \\
29.54 \mathrm{~m}\end{array}$ & $\begin{array}{l}157 \mathrm{~m}^{2} \\
176 \mathrm{~m}^{2}\end{array}$ & $1 \times 10^{\prime}$ & $\begin{array}{l}\text { Offside rule } \\
\text { was not } \\
\text { applied }\end{array}$ & $\begin{array}{l}\text { Ball possession } \\
\text { Players involved } \\
\text { Ball touches } \\
\text { Passes } \\
\text { Shots }\end{array}$ & $\begin{array}{l}\text { Highly skilled players had } \\
\text { greater offensive dynamics } \\
\text { and efficacy }\end{array}$ & 86.7 \\
\hline [11] & $\begin{array}{c}27 \text { soccer } \\
\text { players, } \\
17.4 \pm 0.9 \text { yo }\end{array}$ & $\begin{array}{l}\text { Analyse the } \\
\text { contributions } \\
\text { of physical } \\
\text { performance } \\
\text { and technical } \\
\text { skills on technical } \\
\text { level during SSGs }\end{array}$ & 5 vs. 5 & $30 \times 25 \mathrm{~m}$ & $75 \mathrm{~m}^{2}$ & $4 \times 5^{\prime} / 3^{\prime}$ & $\begin{array}{l}\text { No GK. } \\
\text { Small goals } \\
\text { were used }\end{array}$ & $\begin{array}{l}\text { Players were evaluated } \\
\text { by a game technical } \\
\text { scoring chart in the } \\
\text { following elements: } \\
\text { First touch } \\
\text { Awareness and overall } \\
\text { control } \\
\text { Control from the air } \\
\text { Short passing } \\
\text { Long passing } \\
\text { Dribbling } \\
\text { Turning } \\
\text { Shooting } \\
\text { Accuracy } \\
\text { Two-footedness } \\
\text { Attitude }\end{array}$ & $\begin{array}{l}\text { The best determinant of the } \\
\text { player's technical level during } \\
\text { SSG was the dribbling skill } \\
\text { performed with a pass. } \\
\text { Technical performance was } \\
\text { not meaningfully associated } \\
\text { with physical performance }\end{array}$ & 93.3 \\
\hline [9] & $\begin{array}{c}20 \text { soccer } \\
\text { players, } \\
17.8 \pm 1.0 \text { yo }\end{array}$ & $\begin{array}{l}\text { Analyse } \\
\text { the impact } \\
\text { of mental } \\
\text { fatigue } \\
\text { on technical } \\
\text { performance }\end{array}$ & 5 vs. 5 & $30 \times 20 \mathrm{~m}$ & $60 \mathrm{~m}^{2}$ & $2 \times 7^{\prime} / 1^{\prime}$ & $\begin{array}{l}\text { No GK. } \\
\text { The goal } \\
\text { was to keep } \\
\text { the ball } \\
\text { as long } \\
\text { as possible }\end{array}$ & $\begin{array}{l}\text { Passes } \\
\text { Tackles } \\
\text { Control errors } \\
\text { Possessions } \\
\text { Involvements } \\
\text { Possession time }\end{array}$ & $\begin{array}{l}\text { Mental fatigue negatively } \\
\text { affected technical perfor- } \\
\text { mance. Tackle success, } \\
\% \text { possessions and \% involve- } \\
\text { ments meaningfully decreased } \\
\text { mental fatigue conditions. } \\
\text { Control errors meaningfully } \\
\text { increased under mental fatigue }\end{array}$ & 86.7 \\
\hline
\end{tabular}

yo - years old, GK - goalkeeper, SGG - small-sided game 
Table 5. Effects of training regimens or structured training programs on technical actions and technical performance

\begin{tabular}{|c|c|c|c|c|c|c|c|c|c|c|}
\hline 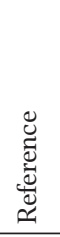 & 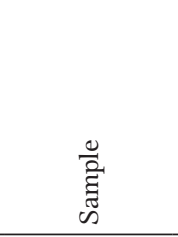 & 寻 & 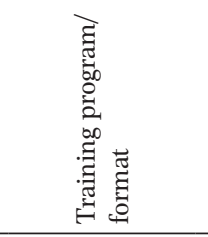 & 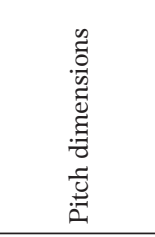 & 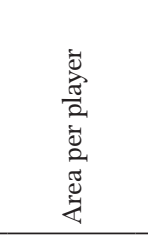 & 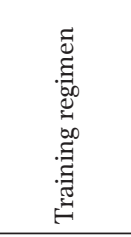 & $\frac{0}{3}$ & 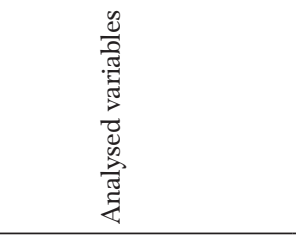 & 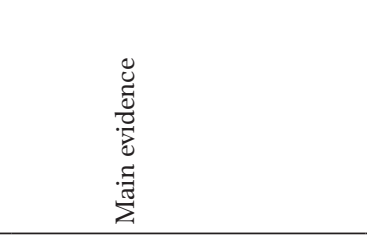 & 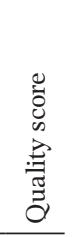 \\
\hline [52] & $\begin{array}{c}20 \text { soccer } \\
\text { players, } \\
15.1 \pm 0.7 \text { yo } \\
(n=11) \\
15.0 \pm 0.5 \text { yo } \\
(n=9)\end{array}$ & $\begin{array}{l}\text { Analyse } \\
\text { the effects } \\
\text { of running- } \\
\text { based high- } \\
\text { intensity } \\
\text { interval } \\
\text { training } \\
\text { and SSGs- } \\
\text { based training } \\
\text { on technical } \\
\text { skills }\end{array}$ & $\begin{array}{l}\text { Running-based } \\
\text { group: } 2 \text { times/ } \\
\text { week for } \\
8 \text { weeks } \\
\\
\text { SSGs group: } \\
3 \text { vs. } 3 \text { or } 3 \text { vs. } 3 \\
+ \text { neutral player } \\
2 \text { times/week } \\
\text { for } 8 \text { weeks }\end{array}$ & $\begin{array}{c}\text { SSGs: } 30 \times \\
18 \mathrm{~m}\end{array}$ & $\begin{array}{c}\text { SSGs: } 90 \\
\mathrm{~m}^{2} \text { and } 77 \\
\mathrm{~m}^{2}\end{array}$ & $5 \times 4^{\prime} / 3^{\prime}$ & Not mentioned & $\begin{array}{l}\text { Technical skills } \\
\text { assessed by a battery } \\
\text { of tests of the German } \\
\text { Soccer Federation } \\
\text { including juggling, } \\
\text { dribbling, heading, } \\
\text { passing the ball into } \\
\text { specified areas, shooting } \\
\text { into specified sectors } \\
\text { of the goal from the kick } \\
\text { point, rotation pass from } \\
\text { the corner of penalty } \\
\text { area, and passing the } \\
\text { ball against the bench }\end{array}$ & $\begin{array}{l}\text { Both groups improved } \\
\text { between pre- and post-training } \\
\text { program. No meaningful } \\
\text { differences between groups } \\
\text { were found }\end{array}$ & 93.8 \\
\hline [49] & $\begin{array}{c}12 \text { soccer } \\
\text { players, } \\
15.4 \pm 0.5 \text { yo }\end{array}$ & $\begin{array}{l}\text { Analyse } \\
\text { the effects } \\
\text { of different } \\
\text { recovery } \\
\text { durations } \\
\text { between bouts } \\
\text { on technical } \\
\text { actions }\end{array}$ & 3 vs. 3 & $30 \times 18 \mathrm{~m}$ & $90 \mathrm{~m}^{2}$ & $\begin{array}{l}4 \times 4^{\prime} / 1^{\prime} \\
4 \times 4^{\prime} / 2^{\prime} \\
4 \times 4^{\prime} / 3^{\prime} \\
4 \times 4^{\prime} / 4^{\prime}\end{array}$ & $\begin{array}{l}\text { Keep ball } \\
\text { possession } \\
\text { as long as } \\
\text { possible. } \\
\text { No goals }\end{array}$ & $\begin{array}{l}\text { Touches of the ball } \\
\text { per possession } \\
\text { Total passes } \\
\text { Successful passes } \\
\text { Tackles } \\
\text { Passes received }\end{array}$ & $\begin{array}{l}\text { Significantly greater values } \\
\text { of total passes and successful } \\
\text { passes were found in the longer } \\
\text { period of recovery (4'). } \\
\text { Significantly more tackles } \\
\text { and passes received occurred } \\
\text { in the 3' recovery period }\end{array}$ & 86.7 \\
\hline [50] & $\begin{array}{c}12 \text { soccer } \\
\text { players, } \\
15.8 \pm 0.6 \text { yo }\end{array}$ & $\begin{array}{l}\text { Analyse } \\
\text { the effects } \\
\text { of different } \\
\text { work-to-rest } \\
\text { ratios on } \\
\text { technical } \\
\text { actions }\end{array}$ & 6 vs. $6+\mathrm{GK}$ & $50 \times 32 \mathrm{~m}$ & $133 \mathrm{~m}^{2}$ & $\begin{array}{l}1 \times 8^{\prime}, \\
2 \times 4^{\prime} / 1^{\prime} \\
4 \times 2^{\prime} / 1^{\prime}\end{array}$ & No offside rule & $\begin{array}{l}\text { Passes } \\
\text { Successful passes } \\
\text { Unsuccessful passes } \\
\text { Shots } \\
\text { Shots on target } \\
\text { Goals } \\
\text { Individual possessions } \\
\text { Regains }\end{array}$ & $\begin{array}{l}\text { Significantly fewer goals } \\
\text { were scored in the continuous } \\
\text { regimen. No significant diffe- } \\
\text { rences were found in the } \\
\text { remaining technical actions }\end{array}$ & 86.7 \\
\hline [51] & $\begin{array}{c}18 \text { soccer } \\
\text { players, } \\
21.8 \pm 4.8 \text { yo } \\
\text { (amateurs) }\end{array}$ & $\begin{array}{l}\text { Analyse } \\
\text { the effects } \\
\text { of running- } \\
\text { based interval } \\
\text { training and } \\
\text { SSGs-based } \\
\text { training on } \\
\text { technical skills }\end{array}$ & $\begin{array}{l}\text { 6-week training } \\
\text { program } \\
\text { (2 times/week) } \\
\text { Running-based } \\
\text { group } \\
\\
\text { SSG-based } \\
\text { program: } \\
5 \text { vs. } 5 \\
6 \text { vs. } 6\end{array}$ & $\begin{array}{l}42 \times 30 \mathrm{~m} \\
\quad(5 \text { vs. } 5) \\
48 \times 36 \mathrm{~m} \\
\quad(6 \text { vs. } 6)\end{array}$ & $\begin{array}{l}126 \mathrm{~m}^{2} \\
144 \mathrm{~m}^{2}\end{array}$ & $\begin{array}{l}\text { Running- } \\
\text { based: } \\
5 \times 6^{\prime} / 3 \text {, } \\
\text { at lactate } \\
\text { threshold } \\
\\
\text { SSGs: } \\
5 \times 6^{\prime} / 3\end{array}$ & Not mentioned & $\begin{array}{l}\text { Technical skills } \\
\text { assessed by Lough- } \\
\text { borough Soccer } \\
\text { Passing Test and } \\
\text { offensive and } \\
\text { defensive skills } \\
\text { measured at match: } \\
\text { Pass } \\
\text { Dribble } \\
\text { Ball control } \\
\text { Tackle } \\
\text { Interception } \\
\text { Aerial duels }\end{array}$ & $\begin{array}{l}\text { SSGs-based group } \\
\text { meaningfully decreased the } \\
\text { time to complete the Lough- } \\
\text { borough Soccer Passing Test } \\
\text { and also increased the number } \\
\text { of defensive and offensive skills } \\
\text { (pre-post analysis). No meaning- } \\
\text { ful changes were found in the } \\
\text { running-based group }\end{array}$ & $\begin{array}{l}86.7 \\
\text { r } \\
\text { s } \\
- \\
-\end{array}$ \\
\hline [53] & $\begin{array}{c}19 \text { soccer } \\
\text { players, } \\
10.6 \pm 0.6 \text { yo } \\
\text { (average-skill } \\
\text { group; } n= \\
\text { 10), } 10.7 \pm \\
0.5 \text { yo (low- } \\
\text { skill group; } n \\
=9 \text { ) }\end{array}$ & $\begin{array}{l}\text { Analyse } \\
\text { the effects of } \\
2 \text { SSG-based } \\
\text { teaching } \\
\text { programs } \\
\text { on decision- } \\
\text { making } \\
\text { and technical } \\
\text { actions }\end{array}$ & $\begin{array}{l}\text { Two periods } \\
\text { of } 14 \text { sessions } \\
\text { of intervention } \\
\text { Intervention 1: } \\
\text { modified games } \\
\text { in numerical } \\
\text { superiority } \\
\text { Intervention 2: } \\
\text { modified games } \\
\text { in numerical } \\
\text { equality }\end{array}$ & $\begin{array}{l}\text { Numerical } \\
\text { superiority: } \\
3 \text { vs. } 2 \\
4 \text { vs. } 3 \\
5 \text { vs. } 4 \\
4 \text { vs. } 4+1 \\
\\
\text { Numerical } \\
\text { equality: } \\
3 \text { vs. } 3 \\
4 \text { vs. } 4 \\
5 \text { vs. } 5\end{array}$ & $\begin{array}{l}30 \times 15 \mathrm{~m} \\
(3 \text { vs. } 2) \\
35 \times 20 \mathrm{~m} \\
(4 \text { vs. } 3) \\
40 \times 25 \mathrm{~m} \\
(5 \text { vs. } 4) \\
\text { Not } \\
\text { mentioned } \\
\text { to } \\
\text { numerical } \\
\text { equality }\end{array}$ & $\begin{array}{c}1 \times 15^{\prime} \\
\text { each }\end{array}$ & $\begin{array}{l}\text { Formats had } \\
\text { different task } \\
\text { conditions based } \\
\text { on the principles } \\
\text { of representation, } \\
\text { complexity, and } \\
\text { exaggeration }\end{array}$ & $\begin{array}{l}\text { Decision-making } \\
\text { and execution } \\
\text { of passes }\end{array}$ & $\begin{array}{l}\text { Numerical superiority led } \\
\text { to meaningful increases } \\
\text { in the decision-making and } \\
\text { pass execution. Low-skill } \\
\text { group also improved the pass } \\
\text { execution from the first } \\
\text { to the second interventions }\end{array}$ & 87.5 \\
\hline
\end{tabular}

yo - years old, GK - goalkeeper, SGG - small-sided game 


\section{Effects of different task conditions}

Two studies [38, 39] tested the effects of different limitations on consecutive ball touches. Three papers [40-42] compared the effects of different scoring methods. Two studies [43, 44] compared the effects of strategic/tactical instructions or constraints. One study [45] assessed the effects of different pitch materials on technical actions performed. One article [46] referred to the effects of different rules (Table 3).

Effects of age group, experience, and technical skill

One study [47] tested the effects of different age groups and expertise levels on technical actions performed during SSGs. One study [48] investigated the effects of different tactical skills. Another paper [11] focused on the effects of different physical performance levels and technical skills on technical actions. Finally, 1 study [9] assessed the impact of players' mental fatigue (Table 4).

\section{Effects of training regimens or structured training programs}

Two studies $[49,50]$ tested the effects of different recovery periods and work-to-rest ratios on technical actions performed by players. Three studies [51-53] evaluated the effects of SSG-based training programs on technical actions and technical skills of players (Table 5).

\section{Discussion}

This systematic review is intended to describe noteworthy evidence about different task constraints on technical actions performed during SSGs. Most of the studies reveal that different conditions influence the frequencies and success of technical actions. The discussion aims to present the main evidence, which is organized in the topics studied.

\section{Effects of format}

Changing the number of players involved in SSGs is a constraint often used by coaches and constitutes one of the main topics researched [1,2]. Typically, SSG formats can be classified as extreme (1 vs. 1$)$, small ( 2 vs. 2 to 4 vs. 4 ), medium ( 5 vs. 5 to 8 vs. 8 ) and large (9 vs. 9 to 11 vs. 11) sided games [25]. The format of play may be balanced (i.e., both teams have the same number of players) or unbalanced (i.e., additional neutral players, i.e. floaters, provide a temporary advantage to the team with possession of the ball). The studies that tested and compared different balanced formats provide different evidence, as the comparisons between formats were different (Table 1). When small-to-medium-sided games (e.g., 2 vs. 2 to 4 vs. 4) were compared with medium-to-large-sided games (i.e., 5 vs. 5 to 11 vs. 11), it was consistently found that a meaningfully greater number of passes, ball contacts, involvements, dribbles, and shots occurred in the small-to-medium games [20, 24-26].

Such evidence was found in very young players (under-12), as well as professional players. An exception to this tendency occurred in a study that compared a small format (3 vs. 3) with a medium format (6 vs. 6) in under-11 players [23]. However, in that study, the relative area per player was not maintained between formats - the 6 vs. 6 format had almost double the relative area per player $\left(195 \mathrm{~m}^{2}\right)$ that the $3 \mathrm{vs}$. 3 format had $\left(98 \mathrm{~m}^{2}\right)$. Naturally, a greater amount of relative space could provide more time and space for players to execute passes and other actions. Nevertheless, it is expectable that players will execute more individual actions in smaller formats than in larger formats, specifically considering that players will have fewer teammates to rely on and that the need to be active and participate in all moments of the match will be higher.

Comparisons among different small formats were also conducted [19, 21, 22]. In this specific case, the evidence is not so clear as it is when small formats are compared with large ones. In a study that compared 3 vs. 3,4 vs. 4 , and 5 vs. 5 formats in under-14 players, no significant changes in terms of technical actions were observed. However, the comparisons could be influenced by the relative pitch area per player [19].

An interesting finding is related to the variability associated with technical actions across games (6.8$19.3 \%$ coefficient of variation) [19]. This is one of the concerns related to SSGs in youth, considering that recent studies have also revealed similarly considerable inter- and intra-session variability among youth players [5, 54]. Also, in studies conducted in under-10 players [22] and professional players [21], small SSGs were associated with meaningful changes in determinant actions, such as passes, receptions, and possessions.

Only 2 studies [27, 28] included in this systematic review tested the effects of unbalanced formats 
of play. A study conducted among amateur and professional players compared differences that were caused by an addition or subtraction of 1 floater (4 vs. 3 and 4 vs. 5) or 3 floaters ( 4 vs. 7). The results reveal that increasing the number of opponents led to a decrease in the number of passes and drives by players with possession [27]. Another study that tested the influence of floaters (positioned inside and outside of the pitch), conducted in professional players [28], reported that floaters contributed to a decrease in the number of dribbles within the pitch, though adding to collective success.

In short, the evidence about different formats reveals that using small formats ( 2 vs. 2 to 4 vs. 4 ) significantly increases the number of determinant technical actions (e.g., passes, receptions, involvements, and dribbles). However, comparisons within categories (e.g., small-, medium-, and large-sided games) are not so clear in terms of meaningful effects on technical actions. Moreover, the use of unbalanced formats contributes to meaningful changes in the technical actions performed. Specifically, the use of floaters results in more successful actions and a higher frequency of defensive actions, although aggressive actions (i.e., dribbles and duels) are reduced.

Effects of pitch size, relative area per player, pitch restrictions and configurations

Different pitch sizes were used for the same format of play to explore the effects of having more or less space and time to make decisions and execute actions depending on the demands of the game. In the present systematic review, 9 studies were included that tested the effects of different pitch sizes (and amounts of relative area per player) or pitch restrictions/configurations. Of these, 5 studies [29-31, 33, 34] investigated the effects of different pitch sizes in the 5 vs. 5 + goalkeeper (GK) format. Among the different amounts of relative area per player (which is calculated as the pitch size divided by the number of players involved, excluding goalkeepers) proposed for the 5 vs. 5 format, the smallest was $40 \mathrm{~m}^{2}(20 \times$ $20 \mathrm{~m})$ [33], and the largest was $273 \mathrm{~m}^{2}(62 \times 44 \mathrm{~m})$ [30, 34].

The findings in 5 vs. 5 SSGs were consistent in that they revealed that smaller pitches $\left(40-101 \mathrm{~m}^{2}\right)$ meaningfully contributed to more tackles [29, 33], dribbles [30, 33], passes/ball possessions [31, 34], and interceptions [30]. These observations suggest that smaller pitches lead to smaller distances between players and, consequently, greater pressure from op- ponents, thus increasing the need to be involved with and to recruit teammates to complete actions and providing more and greater opportunities for players to take defensive actions. Interestingly, in a study conducted among 148 under-12 soccer players, longer ball possessions and fewer transitions occurred on a large pitch $\left(320 \mathrm{~m}^{2}\right)$ than on a small pitch $\left(120 \mathrm{~m}^{2}\right)$ for a 4 vs. 4 + GK format [35].

Changes in the width and length of the pitch can also affect technical actions. A study performed in under-15 players tested the effects of 2 different pitch configurations $(40 \times 30 \mathrm{~m}$ and $30 \times 40 \mathrm{~m})$ with the same relative area per player [36]. The findings reveal that more shots were recorded during games played on the 30-m long field and that more passes occurred in games played on the 40-m long field [36]. These observations suggest that short fields are associated with more shots, considering the reduced distance to the goal, while longer fields increase the need to pass the ball forward before a successful shot can be taken.

The way coaches mark the pitch can also affect the technical actions performed; the hypothesis was tested in the 5 vs. $5+$ GK format on a $30 \times 25 \mathrm{~m}$ pitch $\left(75 \mathrm{~m}^{2}\right.$ per player) [37]. Three different external markings were used (complete lines, dashed lines, and painted markers in the corners). The use of complete and dashed lines resulted in a meaningfully greater number of offensive technical indicators than the use of markers in the corners, although fewer passes were made when complete and dashed lines were used [37].

To summarize the evidence, a small relative area per player $\left(<100 \mathrm{~m}^{2}\right)$ could significantly increase instances of most of the technical actions made by players. However, if the objective of the game is to increase ball possession, very large pitches $(>300$ $\mathrm{m}^{2}$ ) are recommended. Moreover, the length-to-width ratio should be considered. Implementing SSGs on relatively short fields could increase the exploration of attacking finalization (shots and goals), while using longer fields may lead to more passes and longer ball possession times. Finally, it is recommended that markers at the corners of the pitch are used when the exercise is designed to increase the number of passes. Meanwhile, lines are helpful when the goal of training is to increase other attacking actions.

\section{Effects of different task conditions}

Task conditions using modified rules are very common in practical scenarios and in SSG research [1, 2]. The studies included in the present systematic re- 
F. Clemente, H. Sarmento, Small-sided games and technical actions: a review

view examined limitations in terms of the number of consecutive touches of the ball permitted [38, 39], different ways of scoring [40-42], strategic/tactical instructions or constraints [43, 44], different pitch materials [45], and different rules [46] and their effects on technical actions.

The effects of limiting the permitted number of consecutive touches of the ball were tested in 2 vs. 2 , 3 vs. 3 [39], and 4 vs. 4 formats [38]. Comparisons between 1- and 2-touch limitations and free play in a 4 vs. 4 format in professional players revealed that free play increased the number of duels in each bout and decreased the number of technical actions (successful passes and ball losses) [39]. However, in the same study, the 1-touch limitation significantly increased the total number of ball possessions, though the percentage of successful passes decreased significantly [39]. In a comparison of the same ball-touch limitations in different formats ( 2 vs. 2,3 vs. 3 , and 4 vs. 4 ) among professional players, the free-play condition led to the largest number of duels per minute in the 4 vs. 4 format. However, for the 2 vs. 2 format, the largest number of duels occurred under the 2-touch condition [38]. Additionally, the 1-touch limitation significantly decreased the number of successful passes and significantly increased the number of balls lost and the number of possessions.

Both studies [38, 39] tested the effects of ball-touch limitations in professional players. The extent of the decreases in successful actions might be greater for amateur or youth players, and this should be carefully considered. By considering the few studies in professionals, it is possible to identify that free play provides more opportunities than other forms of play to increase the number of duels, while using a 1-touch limitation significantly increases the number of possessions and passes while also contributing to a greater number of errors and balls lost.

Changes to the scoring method are also a common modification made to SSGs [1]. In a study conducted in amateur soccer players using 2 vs. $2+2$ to 4 vs. $4+2$ formats, researchers assessed the effects of different scoring methods, i.e., scoring by crossing the end line (no goal), using 2 small goals located at the end lines, and using a central small goal [40]. Comparisons between scoring methods revealed that attacking actions were performed with greater efficacy in the no-goal condition than in the other 2 conditions [40]. Also, in testing different scoring methods, a study conducted in under-20 players compared the effects of using small goals, goalkeepers, or floaters in different 7 vs. 7 formats [41]. In that study, it was found that using small goals and floaters significantly increased the duration of ball possession when compared with games with goalkeepers [41]. Finally, a study comparing 2-goal games, 4-goal games, 2-goal games with goalposts, and 4-goal games with one goal positioned infield at each corner in a 4 vs. 4 format among under-13 players [42] revealed that more successful forward, backward, and penetrating passes occurred in the 2-goal condition. However, successful sideways passes and turns took place more often in the 4-goal game [42].

In summary, it is possible that the use of goalkeepers reduces the success of passes and ball possession duration and that using no goals or small goals may increase the efficacy of technical actions and increase the frequencies of technical actions related to passes and ball possession. However, it is also important to consider that having goals on the sides will increase the number of sideways passes and turns, while using small goals only on the end lines will increase forward and penetrating passes.

Similarly to a study that tested the effects of coaches' verbal encouragements on physiological responses [55], one of the included studies investigated the effects of providing or not providing instructions for defensive and offensive strategies during a 7 vs. $7+$ GK format among semi-professional players [44]. The results reveal that, when compared with the condition in which no instructions were provided, providing defensive instructions meaningfully increased the frequency of recovered balls and decreased the amount of space occupied, while providing offensive instructions meaningfully increased the number of passes and the amount of space occupied [44]. This suggests that receiving verbal instructions before SSGs can maximize the player's perception for specific behaviours. However, more research should be conducted to test such a hypothesis at different levels of competition and expertise.

Also included in this topic is a study that tested the influence of the type of pitch material (sand or artificial turf) on the number of technical actions made by amateur players during 4 vs. 4 SSGs [45]. The results imply that the use of artificial turf increased the success of technical actions when compared with sand. However, more lifting actions related to the ball were recorded when the SSGs were played on sand.

Effects of age group, experience, and technical skill

Possible factors influencing technical actions and success within SSGs are age, experience, technical 
skills, and tactical knowledge [56, 57]. Although only a few studies were dedicated to examining the effects of such characteristics on technical actions, it was observed in under-13 players that the group of experienced players made longer offensive sequences, with more players executing more touches on the ball and more passes, than inexperienced players [47]. It was also observed in the same study that non-experienced players opted more often for building attacks based on individual actions, while using ball possession was preferred by experienced players [47].

Comparisons between players of different ages and technical skill levels in 3 vs. $3+3+$ GK and 4 vs. $4+$ GK formats revealed that players classified as having more technical skills achieved task goals more often and that those with greater tactical skills exhibited faster pass exchanges [48]. In the same study, it was also found that older players (under-17) presented more individual interventions and shots than younger players (under-15) [48]. Also, when testing the influence of the technical skills and fitness status of under-17 players in a 5 vs. 5 format, it was observed that the best determinant of the technical actions performed by the players was the dribbling skill carried out with a pass. It was also reported that fitness made small contributions to technical actions [11].

Despite the presence of only a few studies on this topic, it can be suggested that more skilled players tend to exhibit more involvement and success regarding technical actions made in small and medium SSGs. Moreover, older players tend to perform more technical actions than younger players.

Another study tested the effects of mental fatigue on players. Not directly referred to in the other articles included in this section, interestingly, mental fatigue negatively affected technical performance during 5 vs. 5 SSGs in under-18 players [9]. In fact, mental fatigue contributed to decreases in successful tackles, in ball possessions percentage, and in percentage of involvements; it also increased control errors [9]. This should be considered, namely in situations in which learning or developing specific technical actions are the primary focus of the drill.

\section{Effects of training regimens or structured training programs}

The use of SSGs in structured training programs has been studied mainly to describe physical and physiological adaptations [58, 59]. However, a few studies tested the effects of structured SSG-based programs on technical skills [51-53]. Two of the included studies
[51, 52] on this topic (Table 5) compared the effects of SSG-based programs and running-based programs.

A study conducted in under-16 players over 8 weeks ( 2 sessions/week; $5 \times 4$ min per session) comparing high-intensity running-based training and interval training using 3 vs. 3 formats on small pitches (77$90 \mathrm{~m}^{2}$ ) revealed that both groups improved in terms of technical skills after the program, without differences between groups [52]. In the other study, which compared high-intensity running-based training and interval SSGs (5 vs. 5 to 6 vs. 6 ) in senior amateur players, it was found that 6 weeks of training ( 2 sessions/week; $5 \times 6 \mathrm{~min}$ per session) were enough for the SSG-based group to meaningfully decrease the time needed to complete the Loughborough Soccer Passing Test and to increase the number of defensive and offensive skills, which was not observed in players who completed the running-based program [51]. Although improvements were expected to be seen in the SSG-based group, neither of the 2 mentioned studies $[51,52]$ provided information about the type of training that players were exposed to before the study or about the trainability level of the players. Thus, these could be confounded factors.

A different approach was applied in a study conducted in under-11 players in which 2 SSG-based protocols were tested [53]. One of the protocols was based on numerical superiority formats ( 3 vs. 2 to 5 vs. 4 ), and the other was based on balanced formats (3 vs. 3 to 5 vs. 5). Both formats included 14 training sessions of $15 \mathrm{~min}$ [53]. The results suggest that greater improvements in decision-making and pass execution occurred in the numerical superiority SSG-based program. However, the study tested 2 groups (low- and average-skill levels), and the low-skill level players also improved in balanced formats, thus indicating that trainability played an important role in the acquisition and consolidation of processes [53].

The type of training regimen can also be manipulated to manage recovery within sessions and to avoid the effects of fatigue on technical actions performed by players [60]. With this assumption, 2 studies [49, 50] tested the effects of different SSG-related work and recovery periods on technical actions. The study conducted in under-16 players investigated the effects of different recovery periods $(1,2,3$, and $4 \mathrm{~min})$ on technical actions performed in a 3 vs. 3 format played over 4 sets of $4 \mathrm{~min}$ [49]. The findings reveal that meaningfully greater values of total passes and successful passes were observed when a longer period of recovery was provided, although more tackles and received passes occurred with the 3-min period of recovery [49]. 
F. Clemente, H. Sarmento, Small-sided games and technical actions: a review

The duration of work repetitions $(1 \times 8$ min vs. $2 \times 4$ min vs. $4 \times 2$ min) was tested by using 6 vs. 6 SSGs in a study conducted in under-16 players. The results show that significantly fewer goals were scored during the continuous regimen, although no other significant changes were found [50].

In summary, despite the existence of only a few studies on this issue, it can be suggested that recovery may affect the capacity of players to perform technical actions more often in small formats played with an interval regimen. Longer periods of recovery and a more balanced work-to-rest ratio might contribute to a high level of technical actions and success. On the other hand, the duration of repetitions may not be essential in large-sided games. Considering the training programs based on SSGs, it is expectable that low-skilllevel players will benefit more from these programs than players at advanced levels. However, it is also expectable that SSG-based programs will provide more benefits than running-based programs when technical skills are considered exclusively.

\section{Future studies}

Despite evidence revealed in this systematic review, there is a lack of consistency in the results and, more important, in the methodologies. Future studies should largely increase the samples and, moreover, conduct repeated analysis with more frequency aiming to avoid the acute effects of learning curve to the game's dynamics and also to identify the variability of actions and behaviours during the period of analysis. Besides, the study of technical actions should be completed in the future by a tactical analysis aiming to check the dependent relationships between both of them. Finally, it is important to consider the intra-game effects of different skill levels in the same match and use some covariables as maturation, tactical knowledge, or others that may be closely related to the variations of technical actions.

\section{Conclusions}

The current systematic review allowed us to summarize the main effects of different task conditions on the technical actions performed by soccer players of different age groups and expertise levels. Some limitations exist regarding the topics considered and the consistency of the methodological approaches used in the examined studies. Nevertheless, it is possible to conclude that small formats ( 2 vs. 2 to 4 vs. 4 ) meaningfully increase the number of determinant technical actions performed (e.g., passes, receptions, involve- ments, and dribbles) when compared with medium and large formats (5 vs. 5 to 11 vs. 11). Moreover, smaller amounts of relative area per player $\left(<100 \mathrm{~m}^{2}\right)$ meaningfully increase most technical actions made by players. However, if the objective of the game is to increase ball possession, very large pitches $\left(>300 \mathrm{~m}^{2}\right)$ are recommended. The use of free play raises the number of technical actions taken, while imposing a 1-touch limitation increases the number of involvements but also the frequencies of errors and balls lost. The use of goalkeepers reduces the number of technical actions performed when compared with the use of small goals. Moreover, the use of goals on the sides and increases in the length of the field raise the number of sideways passes made. It is also important to highlight that providing verbal instructions about defensive and attacking strategies may significantly increase the number of technical actions performed during SSGs. Comparisons between players of different age groups and expertise levels revealed meaningful differences in the frequency and accuracy of technical actions. Thus, such characteristics should be considered. Finally, it was found that allowing greater periods of recovery between bouts might significantly increase the accuracy and frequency of technical actions during SSGs.

\section{Acknowledgments}

FMC: This work is funded by FCT/MCTES through national funds and when applicable co-funded by EU funds under the project UIDB/EEA/50008/2020.

HS gratefully acknowledges the support of the Spanish government subproject 'Integration ways between qualitative and quantitative data, multiple case development, and synthesis review as main axis for an innovative future in physical activity and sports research' (PGC2018-098742-B-C31) (Ministerio de Economía y Competitividad, Programa Estatal de Generación de Conocimiento y Fortalecimiento Científico y Tecnológico del Sistema $\mathrm{I}+\mathrm{D}+\mathrm{i}$ ), that is part of the coordinated project 'New approach of research in physical activity and sport from mixed methods perspective' (NARPAS_MM) [SPGC201800X098742CV0].

\section{Disclosure statement}

No author has any financial interest or received any financial benefit from this research.

\section{Conflict of interest}

The authors state no conflict of interest. 


\section{References}

1. Sarmento H, Clemente FM, Harper LD, Costa IT da, Owen A, Figueiredo AJ. Small sided games in soccer a systematic review. Int J Perform Anal Sport. 2018; 18(5):693-749; doi:10.1080/24748668.2018.1517288.

2. Clemente FM, Martins FML, Mendes RS. Developing aerobic and anaerobic fitness using small-sided soccer games: methodological proposals. Strength Cond J. 2014;36(3):76-87; doi: 10.1519/SSC.000000000000 0063.

3. Davids K, Araújo D, Correia V, Vilar L. How smallsided and conditioned games enhance acquisition of movement and decision-making skills. Exerc Sport Sci Rev. 2013;41(3):154-161; doi: 10.1097/JES.0b013 e318292f3ec.

4. Lacome M, Simpson BM, Cholley Y, Lambert P, Buchheit M. Small-sided games in elite soccer: does one size fit all? Int J Sports Physiol Perform. 2018;13(5):568576; doi: 10.1123/ijspp.2017-0214.

5. Clemente FM, Chen YS, Bezerra JP, Guiomar J, Lima R. Between-format differences and variability of technical actions during small-sided soccer games played by young players. Hum Mov. 2018;19(5):114-120; doi: 10.5114/hm.2018.83103.

6. Clemente FM, Nikolaidis PT, Van Der Linden CMIN, Silva B. Effects of small-sided soccer games on internal and external load and lower limb power: a pilot study in collegiate players. Hum Mov. 2017;18(1):50-57; doi: 10.1515/humo-2017-0007.

7. Serra-Olivares J, Clemente FM, González-Víllora S. Tactical expertise assessment in youth football using representative tasks. Springerplus. 2016;5(1):1301; doi: 10.1186/s40064-016-2955-1.

8. Tan CWK, Chow JY, Davids K. 'How does TGfU work?': examining the relationship between learning design in TGfU and a nonlinear pedagogy. Phys Educ Sport Pedagog. 2012;17(4):331-348; doi: 10.1080/17408989. 2011.582486.

9. Badin OO, Smith MR, Conte D, Coutts AJ. Mental fatigue: impairment of technical performance in smallsided soccer games. Int J Sports Physiol Perform. 2016; 11(8):1100-1105; doi: 10.1123/ijspp.2015-0710.

10. Silva P, Aguiar P, Duarte R, Davids K, Araújo D, Garganta J. Effects of pitch size and skill level on tactical behaviours of association football players during smallsided and conditioned games. Int J Sport Sci Coach. 2014;9(5):993-1006; doi: 10.1260/1747-9541.9.5.993.

11. Rowat O, Fenner J, Unnithan V. Technical and physical determinants of soccer match-play performance in elite youth soccer players. J Sports Med Phys Fitness. 2017; 57(4):369-379; doi: 10.23736/S0022-4707.16.06093-X.

12. Hill-Haas SV, Dawson BT, Impellizzeri FM, Coutts AJ. Physiology of small-sided games training in football: a systematic review. Sports Med. 2011;41(3):199-220; doi: 10.2165/11539740-000000000-00000.

13. Aguiar M, Botelho G, Lago C, Maças V, Sampaio J. A review on the effects of soccer small-sided games. J Hum
Kinet. 2012;33:103-113; doi: 10.2478/v10078-0120049-x.

14. Ometto L, Vasconcellos FVA, Cunha FA, Teoldo I, Souza CRB, Dutra MB, et al. How manipulating task constraints in small-sided and conditioned games shapes emergence of individual and collective tactical behaviours in football: a systematic review. Int J Sports Sci Coach. 2018;13(6):1200-1214; doi: 10.1177/17479541 18769183.

15. Bujalance-Moreno P, Latorre-Román PÁ, García-Pinillos F. A systematic review on small-sided games in football players: acute and chronic adaptations. J Sports Sci.2019;37(8):921-949; doi:10.1080/02640414.2018. 1535821.

16. Moher D, Liberati A, Tetzlaff J, Altman DG, The PRISMA Group. Preferred reporting items for systematic reviews and meta-analyses: the PRISMA statement. Int J Surg. 2010;8(5):336-341; doi: 10.1016/j.ijsu.2010.02.007.

17. Sarmento H, Clemente FM, Araújo D, Davids K, McRobert A, Figueiredo A. What performance analysts need to know about research trends in association football (2012-2016): a systematic review. Sport Med. 2018; 48(4):799-836; doi: 10.1007/s40279-017-0836-6.

18. Ryan R, Synnot A, Prictor M, Hill S. Cochrane Consumers and Communication Group Data extraction template for included studies. Melbourne: La Trobe University; 2016. Available from: http:/cccrg.cochrane.org/ author-resources.

19. Da Silva CD, Impellizzeri FM, Natali AJ, de Lima JR, Bara-Filho MG, Silami-Garçia E, et al. Exercise intensity and technical demands of small-sided games in young Brazilian soccer players: effect of number of players, maturation, and reliability. J Strength Cond Res. 2011;25(10):2746-2751; doi: 10.1519/JSC.0b013e31820 da061.

20. Owen AL, Wong del P, McKenna M, Dellal A. Heart rate responses and technical comparison between small- vs. large-sided games in elite professional soccer. J Strength Cond Res. 2011;25(8):2104-2110; doi: 10.1519/JSC. 0b013e3181f0a8a3.

21. Dellal A, Drust B, Lago-Penas C. Variation of activity demands in small-sided soccer games. Int J Sports Med. 2012;33(5):370-375; doi: 10.1055/s-0031-1295476.

22. Castelão D, Garganta J, Santos R, Teoldo I. Comparison of tactical behaviour and performance of youth soccer players in 3v3 and 5v5 small-sided games. Int J Perform Anal Sport. 2014;14(3):801-813; doi: 10.1080/ 24748668.2014.11868759.

23. Silva B, Garganta J, Santos R, Teoldo I. Comparing tactical behaviour of soccer players in 3 vs. 3 and 6 vs. 6 small-sided games. J Hum Kinet. 2014;41:191-202; doi: 10.2478/hukin-2014-0047.

24. Dellal A, Owen A, Wong DP, Krustrup P, van Exsel M, Mallo J. Technical and physical demands of small vs. large sided games in relation to playing position in elite soccer. Hum Mov Sci. 2012;31(4):957-969; doi: 10.1016/j.humov.2011.08.013. 
F. Clemente, H. Sarmento, Small-sided games and technical actions: a review

25. Owen AL, Wong DP, Paul D, Dellal A. Physical and technical comparisons between various-sided games within professional soccer. Int J Sports Med. 2014;35(4):286292; doi: 10.1055/s-0033-1351333.

26. Oh SH, Joo CH. Comparison of technical and physical activities between 8 vs. 8 and 11 vs. 11 games in young Korean soccer players. JExerc Rehabil. 2018;14(2):253258; doi: 10.12965/jer.1836034.017.

27. Torrents C, Ric A, Hristovski R, Torres-Ronda L, Vicente E, Sampaio J. Emergence of exploratory, technical and tactical behavior in small-sided soccer games when manipulating the number of teammates and opponents. PLoS One. 2016;11(12):e0168866; doi: 10.1371/journal.pone.0168866.

28. Sanchez-Sanchez J, Hernández D, Casamichana D, Martínez-Salazar C, Ramirez-Campillo R, Sampaio J. Heart rate, technical performance, and session-RPE in elite youth soccer small-sided games played with wildcard players. J Strength Cond Res. 2017;31(10): 2678-2685; doi: 10.1519/JSC.0000000000001736.

29. Kelly DM, Drust B. The effect of pitch dimensions on heart rate responses and technical demands of smallsided soccer games in elite players. J Sci Med Sport. 2009;12(4):475-479; doi: 10.1016/j.jsams.2008.01.010.

30. Casamichana D, Castellano J. Time-motion, heart rate, perceptual and motor behaviour demands in smallsides soccer games: effects of pitch size. J Sports Sci. 2010;28(14):1615-1623; doi: 10.1080/02640414.2010. 521168.

31. Aslan A. Cardiovascular responses, perceived exertion and technical actions during small-sided recreational soccer: effects of pitch size and number of players. J Hum Kinet. 2013;38:95-105; doi: 10.2478/ hukin-2013-0049.

32. Joo CH, Hwang-Bo K, Jee H. Technical and physical activities of small-sided games in young Korean soccer players. J Strength Cond Res. 2016;30(8):2164-2173; doi: 10.1519/JSC.0000000000001319.

33. Martone D, Giacobbe M, Capobianco A, Imperlini E, Mancini A, Capasso M, et al. Exercise intensity and technical demands of small-sided soccer games for under-12 and under-14 players: effect of area per player. J Strength Cond Res. 2017;31(6):1486-1492; doi: 10.1519/JSC. 0000000000001615.

34. Jara D, Ortega E, Gomez MÁ, de Baranda PS. Effect of pitch size on technical-tactical actions of the goalkeeper in small-sided games. J Hum Kinet. 2018;62:157166; doi: 10.1515/hukin-2017-0167.

35. Olthof SBH, Frencken WGP, Lemmink KAPM. Matchderived relative pitch area changes the physical and team tactical performance of elite soccer players in smallsided soccer games. J Sports Sci. 2018;36(14):15571563; doi: 10.1080/02640414.2017.1403412.

36. Folgado H, Bravo J, Pereira P, Sampaio J. Towards the use of multidimensional performance indicators in football small-sided games: the effects of pitch orienta- tion. J Sports Sci. 2019;37(9):1064-1071; doi: 10.1080/ 02640414.2018 .1543834$.

37. Coutinho D, Gonçalves B, Travassos B, Folgado H, Figueira B, Sampaio J. Different marks in the pitch constraint youth players' performances during football small-sided games. Res Q Exerc Sport. 2019;1-9; doi: 10.1080/02701367.2019.1645938.

38. Dellal A, Chamari K, Owen AL, Wong DP, Lago-Penas C, Hill-Haas S. Influence of technical instructions on the physiological and physical demands of smallsided soccer games. Eur J Sport Sci. 2011;11(5):341346; doi: 10.1080/17461391.2010.521584.

39. Dellal A, Lago-Penas C, Wong del P, Chamari K. Effect of the number of ball contacts within bouts of 4 vs. 4 small-sided soccer games. Int J Sports Physiol Perform. 2011;6(3):322-333; doi: 10.1123/ijspp.6.3.322.

40. Clemente FM, Wong del P, Martins FM, Mendes RS. Acute effects of the number of players and scoring method on physiological, physical, and technical performance in small-sided soccer games. Res Sports Med. 2014; 22(4):380-397; doi: 10.1080/15438627.2014.951761.

41. Castellano J, Silva P, Usabiaga O, Barreira D. The influence of scoring targets and outer-floaters on attacking and defending team dispersion, shape and creation of space during small-sided soccer games. J Hum Kinet. 2016;51:153-163; doi: 10.1515/hukin-2015-0178.

42. Pulling C, Twitchen A, Pettefer C. Goal format in smallsided soccer games: technical actions and offensive scenarios of prepubescent players. Sports. 2016;4(4): 53; doi: 10.3390/sports4040053.

43. Abrantes CI, Nunes MI, Maçãs VM, Leite NM, Sampaio JE. Effects of the number of players and game type constraints on heart rate, rating of perceived exertion, and technical actions of small-sided soccer games. J Strength Cond Res. 2012;26(4):976-981; doi: 10.1519/ JSC.0b013e31822dd398.

44. Batista J, Goncalves B, Sampaio J, Castro J, Abade E, Travassos B. The influence of coaches' instruction on technical actions, tactical behaviour, and external workload in football small-sided games. Monten J Sports SciMed. 2019;8(1):29-36; doi: 10.26773/mjssm.190305.

45. Rago V, Rebelo AN, Pizzuto F, Barreira D. Small-sided soccer games on sand are more physically demanding but less technically specific compared to games on artificial turf. J Sports Med Phys Fitness. 2018;58(4):385391; doi: 10.23736/S0022-4707.16.06708-6.

46. Machado JC, Ribeiro J, Palheta CE, Alcântara C, Barreira $\mathrm{D}$, Guilherme J, et al. Changing rules and configurations during soccer small-sided and conditioned games. How does it impact teams' tactical behavior? Front Psychol. 2019;10:1554; doi: 10.3389/fpsyg.2019.01554.

47. Almeida CH, Ferreira AP, Volossovitch A. Offensive sequences in youth soccer: effects of experience and small-sided games. J Hum Kinet. 2013;36:97-106; doi: 10.2478/hukin-2013-0010.

48. Machado JC, Barreira D, Teoldo I, Travassos B, Júnior JB, Dos Santos JOL, et al. How does the adjustment of 
training task difficulty level influence tactical behavior in soccer? Res Q Exerc Sport. 2019;90(3):403-416; doi: 10.1080/02701367.2019.1612511.

49. Köklü Y, Alemdaroğlu U, Dellal A, Wong DP. Effect of different recovery durations between bouts in 3-a-side games on youth soccer players' physiological responses and technical activities. J Sports Med Phys Fitness. 2015;55(5):430-438.

50. Christopher J, Beato M, Hulton AT. Manipulation of exercise to rest ratio within set duration on physical and technical outcomes during small-sided games in elite youth soccer players. Hum Mov Sci. 2016;48:1-6; doi: 10.1016/j.humov.2016.03.013.

51. Özcan İ, Eniseler N, Şahan Ç. Effects of small-sided games and conventional aerobic interval training on various physiological characteristics and defensive and offensive skills used in soccer. Kinesiology. 2018;50(1): 104-111; doi: 10.26582/k.50.1.12.

52. Radziminski L, Rompa P, Barnat W, Dargiewicz R, Jastrzebski Z. A comparison of the physiological and technical effects of high-intensity running and smallsided games in young soccer players. Int J Sports Sci Coach. 2013;8(3):455-465; doi: 10.1260/1747-9541. 8.3.455.

53. Práxedes A, Moreno A, Gil-Arias A, Claver F, Del Villar F. The effect of small-sided games with different levels of opposition on the tactical behaviour of young footballers with different levels of sport expertise. PLoS One. 2018;13(1):e0190157; doi: 10.1371/journal.pone. 0190157.

54. Clemente FM, Sarmento H, Costa IT, Enes AR, Lima R. Variability of technical actions during small-sided games in young soccer players. J Hum Kinet. 2019;69:201212; doi: 10.2478/hukin-2019-0013.

55. Rampinini E, Impellizzeri FM, Castagna C, Abt G, Chamari K, Sassi A, et al. Factors influencing physiological responses to small-sided soccer games. J Sports Sci. 2007;25(6):659-666; doi: 10.1080/02640410600 811858.

56. Praça GM, Costa CLA, Costa FF, de Andrade AGP, Chagas MH, Greco JP. Tactical behavior in soccer smallsided games: influence of tactical knowledge and numerical superiority. J Phys Educ. 2016;27:e2736; doi: 10.4025/jphyseduc.v27i1.2736.

57. Praça GM, Soares VV, Matias CJA da S, da Costa IT, Greco PJ. Relationship between tactical and technical performance in youth soccer players. Rev Bras Cineantropom Desempenho Hum. 2015;17(2):136-144; doi: 10.5007/1980-0037.2015v17n2p136.

58. Owen AL, Wong del P, Paul D, Dellal A. Effects of a periodized small-sided game training intervention on physical performance in elite professional soccer. J Strength Cond Res. 2012;26(10):2748-2754; doi: 10. 1519/JSC.0b013e318242d2d1.

59. Rabbani A, Clemente FM, Kargarfard M, Jahangiri S. Combined small-sided game and high-intensity interval training in soccer players: the effect of exercise order.
J Hum Kinet. 2019;69:249-257; doi: 10.2478/hukin2018-0092.

60. Clemente FM, Nikolaidis PT, Rosemann T, Knechtle B. Variations of internal and external load variables between intermittent small-sided soccer game training regimens. Int J Environ Res Public Health. 2019;16(16): 2923; doi: 10.3390/ijerph16162923. 